\title{
No Infimum Gap and Normality in Optimal Impulsive Control Under State Constraints
}

\author{
Giovanni Fusco ${ }^{1}$ (D) . Monica Motta ${ }^{1}$ (D)
}

Received: 13 July 2020 / Accepted: 4 February 2021 / Published online: 27 April 2021

(C) The Author(s) 2021

\begin{abstract}
In this paper we consider an impulsive extension of an optimal control problem with unbounded controls, subject to endpoint and state constraints. We show that the existence of an extended-sense minimizer that is a normal extremal for a constrained Maximum Principle ensures that there is no gap between the infima of the original problem and of its extension. Furthermore, we translate such relation into verifiable sufficient conditions for normality in the form of constraint and endpoint qualifications. Links between existence of an infimum gap and normality in impulsive control have previously been explored for problems without state constraints. This paper establishes such links in the presence of state constraints and of an additional ordinary control, for locally Lipschitz continuous data.
\end{abstract}

Keywords Impulsive optimal control problems $\cdot$ Maximum principle $\cdot$ State constraints · Gap phenomena $\cdot$ Normality $\cdot$ Degeneracy

Mathematics Subject Classification (2010) $49 \mathrm{~N} 25 \cdot 34 \mathrm{~K} 45 \cdot 49 \mathrm{~K} 15$

\section{Introduction}

In Optimal Control Theory it is quite common practice to extend the domain of a minimum problem to ensure the existence of the minimum or to identify optimality conditions. In doing this, it is of course desirable to avoid the so-called infimum gap phenomenon, i.e. that the minimum of the extended problem is different from the minimum of the original problem. This is relevant not only for theoretical reasons of 'well-posedness' of the

This research is partially supported by the INdAM-GNAMPA Project 2020 "Extended control problems: gap, higher order conditions and Lyapunov functions" and by the Padua University grant SID 2018 "Controllability, stabilizability and infimum gaps for control systems", prot. BIRD 187147.

Monica Motta

motta@math.unipd.it

Giovanni Fusco

fusco@math.unipd.it

1 Department of Mathematics Tullio Levi-Civita, University of Padua, Via Trieste, 63, Padova 35121 , Italy 
extension, but also for the actual usefulness of the extended problem in order to identify, for instance, necessary optimality conditions or a non degenerate Hamilton-Jacobi-Bellman equation for the original minimum problem. For the classical extension of a minimum problem by convex relaxation -where the original velocity set of the trajectories is replaced by its convexification - it has emerged that a sufficient condition to avoid the infimum gap is the normality of an extended sense minimizer, namely, that all sets of multipliers verifying a Maximum Principle have cost multiplier, $\lambda$ in the following, different from zero $[29,30,36,38-40]$. In [26] the 'normality test' has been proved to be sufficient to guarantee the absence of an infimum gap also for the impulsive extension of an optimal control problem with unbounded controls. Very recently, in [27, 28] this link between normality and no-infimum-gap has been established for the extension of an abstract minimum control problem, which includes both relaxation and impulsive extension. All the above results in the case of the impulsive extension concern problems without state constraints, with $C^{1}$ data, and no ordinary controls in the dynamics. However, state constraints, together with nonsmoothness of the data and additional ordinary controls, arise very frequently in the applications of impulsive optimal control (see e.g. [7, 16, 19, 22, 34] and the references therein).

This paper provides 'normality type' sufficient conditions to avoid a gap between the infima of the following optimization problem $(\mathrm{P})$ and the extended optimization problem $\left(\mathrm{P}_{e}\right)$ below:

$$
\text { minimize } \Psi\left(t_{1}, x\left(t_{1}\right), t_{2}, x\left(t_{2}\right), v\left(t_{2}\right)\right)
$$

over $t_{1}, t_{2} \in \mathbb{R}, t_{1}<t_{2}, \quad(x, v, u) \in W^{1,1}\left(\left[t_{1}, t_{2}\right] ; \mathbb{R}^{n+1+m}\right), a \in L^{1}\left(\left[t_{1}, t_{2}\right] ; A\right)$ satisfying

$$
\left\{\begin{array}{l}
\frac{d x}{d t}(t)=f(t, x(t), a(t))+\sum_{j=1}^{m} g_{j}(t, x(t)) \frac{d u^{j}}{d t}(t) \quad \text { a.e. } t \in\left[t_{1}, t_{2}\right], \\
\frac{d v}{d t}(t)=\left|\frac{d u}{d t}(t)\right| \quad \text { a.e. } t \in\left[t_{1}, t_{2}\right], \\
\frac{d u}{d t}(t) \in \mathcal{C} \quad \text { a.e. } t \in\left[t_{1}, t_{2}\right], \\
h_{1}(t, x(t)) \leq 0, \ldots, h_{N}(t, x(t)) \leq 0 \quad \text { for all } t \in\left[t_{1}, t_{2}\right], \\
v\left(t_{1}\right)=0, \quad v\left(t_{2}\right) \leq K, \quad\left(t_{1}, x\left(t_{1}\right), t_{2}, x\left(t_{2}\right)\right) \in \mathcal{T}_{0},
\end{array}\right.
$$

where $K>0$ is a fixed constant (possibly equal to $+\infty$ ), $A \subset \mathbb{R}^{q}$ is a compact subset, $\mathcal{C} \subseteq \mathbb{R}^{m}$ is a closed convex cone, $\mathcal{T}_{0} \subseteq \mathbb{R}^{1+n+1+n}$ is a closed subset, and the data are locally Lipschitz continuous in $t, x$ (the precise assumptions will be given in Section 2). Problem (P) is a free end-time minimization problem depending on an ordinary control $a$ and on a control $u$ whose derivatives appear linearly in the dynamics. Furthermore, there are timedependent state constraints in the form of $N$ inequalities, endpoint constraints, and we may have a bound $K$ on the total variation of $u$-notice that $v$ is nothing but the total variation function of $u$. Due to a lack of coerciveness, minimizers for problem (P) do not exist in general. Hence, adopting a by now standard extension, we embed the original problem into the space-time problem $\left(\mathrm{P}_{e}\right)$ below, where the extended state variable is $\left(y^{0}, y, v\right):=(t, x, v)$, and the extended trajectories are $(t, x, v)$-paths which are (reparameterized) $C^{0}$-limits of graphs of the original trajectories $[10,21,23,32,37]:^{1}$

$$
\text { minimize } \Psi\left(y^{0}(0), y(0), y^{0}(S), y(S), v(S)\right)
$$

\footnotetext{
${ }^{1}$ As it is well-known, a distributional approach, where $\frac{d u}{d t}$ is interpreted as a Radon measure, does not work unless $g_{i}=g_{i}(x)$ and the Lie brackets $\left[g_{i}, g_{j}\right](x) \equiv 0$ for every $i, j=1, \ldots, m$ (see e.g. $[10,15]$ ).
} 
over $S>0, \quad\left(y^{0}, y, v\right) \in W^{1,1}\left([0, S] ; \mathbb{R}^{1+n+1}\right), \quad\left(\omega^{0}, \omega, \alpha\right) \in L^{1}([0, S] ; \mathbf{C} \times A)$ satisfying

$$
\left\{\begin{array}{l}
\frac{d y^{0}}{d s}(s)=\omega^{0}(s) \quad \text { a.e. } s \in[0, S], \\
\frac{d y}{d s}(s)=f\left(y^{0}, y, \alpha\right)(s) \omega^{0}(s)+\sum_{j=1}^{m} g_{j}\left(y^{0}, y\right)(s) \omega^{j}(s) \quad \text { a.e. } s \in[0, S], \\
\frac{d v}{d s}(s)=|\omega(s)| \quad \text { a.e. } s \in[0, S], \\
h_{1}\left(y^{0}(s), y(s)\right) \leq 0, \ldots, h_{N}\left(y^{0}(s), y(s)\right) \leq 0 \quad \text { for all } s \in[0, S], \\
v(0)=0, \quad v(S) \leq K, \quad\left(y^{0}(0), y(0), y^{0}(S), y(S)\right) \in \mathcal{T}_{0},
\end{array}\right.
$$

where $\mathbf{C}:=\left\{\left(\omega^{0}, \omega\right):\left(\omega^{0}, \omega\right) \in\left[0,+\infty\left[\times \mathcal{C}, \omega^{0}+|\omega|=1\right\}\right.\right.$. To any process $\left(t_{1}, t_{2}, u, a, x, v\right)$ of problem (P), by setting $\sigma(t):=t-t_{1}+v(t), t \in\left[t_{1}, t_{2}\right]$, through the time-change $y^{0}:=\sigma^{-1}$ we can associate a process $\left(S, \omega^{0}, \omega, \alpha, y^{0}, y, v\right)$ of the extended problem with $\omega^{0}=d y^{0}(s) / d s>0$ a.e.. In particular, problem (P) can be identified with the restriction of problem $\left(\mathrm{P}_{e}\right)$ to the set of processes with $\omega^{0}>0$ a.e. (see Section 2). Let us refer to such processes as embedded strict sense processes in the following. The extension consists therefore in considering extended sense processes with $\omega^{0}=0$ on non-degenerate intervals, where the time $t=y^{0}(s)$ is constant but the extended state $y(s)$ evolves according to the 'fast' dynamics $d y(s) / d s=\sum_{j=1}^{m} g_{j}\left(y^{0}(s), y(s)\right) \omega^{j}(s)$. This explains why $\left(\mathrm{P}_{e}\right)$ is also called the impulsive problem, although it is a conventional optimization problem with bounded controls. In fact, one could give an equivalent $t$-based description of this extension using bounded variation trajectories and controls [2, 7, 19, 22, 24, 25, 33, 41].

The main result of the paper, obtained in Theorem 4.1 below, establishes that the existence of an extended sense minimizer for problem $\left(\mathrm{P}_{e}\right)$ which is a normal extremal for a constrained version of the Maximum Principle, is a sufficient condition for the infimum gap avoidance. The occurrence of a gap is strictly related to the presence of endpoints and state constraints. In particular, since the set of trajectories corresponding to embedded strict sense processes is $C^{0}$-dense in the set of trajectories of the extended system, the infimum gap phenomenon can show up only when some extended sense process verifying the constraints is isolated, namely cannot be approximated by trajectories of the original system that satisfy the constraints. From this observation, Theorem 4.1 will be derived from a general result on the properties of isolated processes (see Theorem 3.2). The proof makes use of perturbation and penalization techniques and of the Ekeland's variational principle, in the same spirit of $[26,29]$. This approach is very different from that of $[28,38-40]$, which is based on the construction of approximating cones to reachable sets and on set separation arguments. We recall that normality is not necessary to exclude the gap phenomenon: for example, it is known that without the drift $f$ in the dynamics, gap never occurs [26, Lemma 4.1] (see also [20]).

The normality criterium for the absence of an infimum gap has some disadvantages. First of all, it requires to know a priori a minimizing extended sense process, information that is not always available. Then, it is necessary to verify that all sets of multipliers associated to the minimizer that meet the conditions of the Maximum Principle have $\lambda>0$. In addition, in the presence of state constraints the normality condition may never be met, making the criterium in fact useless. In particular, it is well known that when the state constraint is active at the initial point of a minimizing process, sets of degenerate multipliers with $\lambda=0$ may always exist. Rather surprisingly, it seems that no attention has been paid to this 'degeneracy question' in previous articles on the relationship between gap and normality in the presence of state constraints. 
Based on the above considerations, in the second part of the paper we first introduce a nondegenerate version of the Maximum Principle and provide simple geometrical conditions on endpoint and state constraints, under which abnormal -namely, not normalextremals for the original Maximum Principle turn out to be abnormal extremals also for the nondegenerate Maximum Principle. In this case, Theorem 4.1 can be rephrased as follows: 'normality among nondegenerate multipliers implies no infimum gap'. This 'nondegenerate normality test' is useful especially because in certain special cases it allows to deduce the absence of the infimum gap from easily verifiable conditions, some examples of which we will provide. In particular, these are constraint and endpoint qualification conditions.

Although this article is mainly focused on the infimum gap phenomenon, it also establishes some new sufficient conditions for normality which extend previous conditions in [25]. In the literature on conventional, non-impulsive problems with state constraints, a variety of constraint qualifications to avoid degeneracy as well as to ensure normality are known (see e.g. [4, 5, 8, 9, 11-14, 18, 30, 31] and the references therein). In impulsive control, instead, some nondegenerate Maximum Principles have been obtained in [6, 7, 17, 25], while a Maximum Principle in normal form has only recently been introduced in [25].

The paper is organized as follows: in Section 2 we introduce precisely problems $(\mathrm{P}),\left(\mathrm{P}_{\mathrm{e}}\right)$ and a constrained version of the Maximum Principle for the extended problem. Section 3 is devoted to prove that an isolated extended sense extremal cannot be normal and, as a corollary, we deduce that presence of an infimum gap implies abnormality of any extended sense minimizer. In Section 4 we provide sufficient conditions for normality, which guarantee a priori, without any knowledge of the multipliers associated with the given extended sense minimizer, the non occurrence of gap-phenomena. In Section 5, we present some examples to illustrate the results.

\subsection{Notations and Preliminaries}

Given an interval $I \subseteq \mathbb{R}$ and a set $X \subseteq \mathbb{R}^{k}$, we write $C^{0}(I ; X), W^{1,1}(I ; X), C^{0,1}(I ; X)$, $C_{l o c}^{0,1}(I ; X)$ for the space of continuous functions, absolutely continuous functions, Lipschitz continuous functions, locally Lipschitz continuous functions defined on $I$ and with values in $X$, respectively. For all the classes of functions introduced so far, we will not specify domain and codomain when the meaning is clear. Furthermore, we denote by $\ell(X), \operatorname{co}(X)$, $\operatorname{Int}(X), \partial X$ the Lebesgue measure, the convex hull, the interior and the boundary of $X$, respectively. As customary, $\chi_{X}$ is the characteristic function of $X$, namely $\chi_{X}(x)=1$ if $x \in X$ and $\chi_{X}(x)=0$ if $x \in \mathbb{R}^{k} \backslash X ; I \cdot X$ denotes the set $\{r x \mid r \in I, x \in X\}$. Given two nonempty subsets $X_{1}, X_{2}$ of $\mathbb{R}^{k}$, we denote by $X_{1}+X_{2}$ the set $\left\{x_{1}+x_{2} \mid x_{1} \in X_{1}, x_{2} \in X_{2}\right\}$. Let $X \subseteq \mathbb{R}^{k_{1}+k_{2}}$ for some natural numbers $k_{1}, k_{2}$, and write $x=\left(x_{1}, x_{2}\right) \in \mathbb{R}^{k_{1}} \times \mathbb{R}^{k_{2}}$ for any $x \in X$. Then $\operatorname{proj}_{x_{i}} X$ will denote the projection of $X$ on $\mathbb{R}^{k_{i}}$, for $i=1,2$. We denote the closed unit ball in $\mathbb{R}^{k}$ by $\mathbb{B}_{k}$, omitting the dimension when it is clear from the context. Given a closed set $\mathcal{O} \subseteq \mathbb{R}^{k}$ and a point $z \in \mathbb{R}^{k}$, we define the distance of $z$ from $\mathcal{O}$ as $d_{\mathcal{O}}(z):=\min _{y \in \mathcal{O}}|z-y|$. We set $\mathbb{R}_{\geq 0}:=[0,+\infty[$. For any $a, b \in \mathbb{R}$, we write $a \vee b:=\max \{a, b\}$.

For all $\tau_{1}, \tau_{2}, \bar{\tau}_{1}, \bar{\tau}_{2} \in \mathbb{R}, \tau_{1}<\tau_{2}, \bar{\tau}_{1}<\bar{\tau}_{2}$, and for any pair $\left(z_{1}, z_{2}\right) \in C^{0}\left(\left[\tau_{1}, \tau_{2}\right], \mathbb{R}^{k}\right) \times$ $C^{0}\left(\left[\bar{\tau}_{1}, \bar{\tau}_{2}\right], \mathbb{R}^{k}\right)$, let us define the distance

$$
d_{\infty}\left(\left(\tau_{1}, \tau_{2}, z_{1}\right),\left(\bar{\tau}_{1}, \bar{\tau}_{2}, z_{2}\right)\right):=\left|\tau_{1}-\bar{\tau}_{1}\right|+\left|\tau_{2}-\bar{\tau}_{2}\right|+\left\|\tilde{z}_{1}-\tilde{z}_{2}\right\|_{L^{\infty}(\mathbb{R})},
$$

where for any $z \in C^{0}\left([a, b], \mathbb{R}^{k}\right), \tilde{z}$ denotes its continuous constant extension to $\mathbb{R}$ and $\|$. $\|_{L^{\infty}(I)}$ is the ess-sup norm on $I \subseteq \mathbb{R}$ interval. When the domain is clear, we will sometimes simply write $\|\cdot\|_{L^{\infty}}$. 
We denote by $N B V^{+}([0, S] ; \mathbb{R})$ the space of increasing, real valued functions $\mu$ on $[0, S]$ of bounded variation, vanishing at the point 0 and right continuous on $] 0, S[$. Each $\mu \in$ $N B V^{+}([0, S] ; \mathbb{R})$ defines a Borel measure on $[0, S]$, still denoted by $\mu$, its total variation function is indicated by $\|\mu\|_{T V}$ or equivalently by $\mu([0, S])$, and its support by $\operatorname{spt}\{\mu\}$.

Some standard constructs from nonsmooth analysis are employed in this paper. For background material we refer the reader for instance to [35]. A set $K \subseteq \mathbb{R}^{k}$ is a cone if $\alpha k \in K$ for any $\alpha>0$, whenever $k \in K$. Take a closed set $D \subseteq \mathbb{R}^{k}$ and a point $\bar{x} \in D$, the proximal normal cone $N_{D}^{P}(\bar{x})$ of $D$ at $\bar{x}$ is defined as

$$
N_{D}^{P}(\bar{x}):=\left\{\eta \in \mathbb{R}^{k}: \exists M>0 \text { such that } \eta \cdot(x-\bar{x}) \leq M|x-\bar{x}|^{2} \forall x \in D\right\} .
$$

The limiting normal cone $N_{D}(\bar{x})$ of $D$ at $\bar{x}$ is given by

$$
N_{D}(\bar{x}):=\left\{\eta \in \mathbb{R}^{k}: \exists x_{i} \stackrel{D}{\rightarrow} \bar{x}, \eta_{i} \rightarrow \eta \text { s.t. } \eta_{i} \in N_{D}^{P}\left(x_{i}\right) \text { for each } i \in \mathbb{N}\right\},
$$

in which the notation $x_{i} \stackrel{D}{\longrightarrow} \bar{x}$ is used to indicate that all points in the converging sequence $\left(x_{i}\right)_{i \in \mathbb{N}}$ lay in $D$. In general, $N_{D}^{P}(\bar{x}) \subseteq N_{D}(\bar{x})$. Take a lower semicontinuous function $G$ : $\mathbb{R}^{k} \rightarrow \mathbb{R}$ and a point $\bar{x} \in \mathbb{R}^{k}$, the limiting subdifferential of $G$ at $\bar{x}$ is

$$
\partial G(\bar{x}):=\left\{\xi \in \mathbb{R}^{k}:(\xi,-1) \in N_{\text {epi }(G)}(\bar{x}, G(\bar{x}))\right\},
$$

where epi $(G)$ is the epigraph of $G$. If $G: \mathbb{R}^{k} \times \mathbb{R}^{h} \rightarrow \mathbb{R}$ is a lower semicontinuous function and $(\bar{x}, \bar{y}) \in \mathbb{R}^{k} \times \mathbb{R}^{h}$, we write $\partial_{x} G(\bar{x}, \bar{y}), \partial_{y} G(\bar{x}, \bar{y})$ to denote the partial limiting subdifferential of $G$ at $(\bar{x}, \bar{y})$ w.r.t. $x, y$, respectively. Given $G \in C_{l o c}^{0,1}\left(\mathbb{R}^{k} ; \mathbb{R}\right)$ and $\bar{x} \in \mathbb{R}^{k}$, the reachable hybrid subdifferential of $G$ at $\bar{x}$ is

$$
\begin{aligned}
\partial^{*>} G(\bar{x}):=\left\{\xi \in \mathbb{R}^{k}: \exists\left(x_{i}\right)_{i} \subset \operatorname{diff}(G) \backslash\{\bar{x}\}\right. \text { s.t. } \\
\left.x_{i} \rightarrow \bar{x}, \quad G\left(x_{i}\right)>0 \forall i \text { and } \nabla G\left(x_{i}\right) \rightarrow \xi\right\},
\end{aligned}
$$

while the reachable gradient of $G$ at $\bar{x}$ is

$$
\partial^{*} G(\bar{x}):=\left\{\xi \in \mathbb{R}^{k}: \exists\left(x_{i}\right)_{i} \subset \operatorname{diff}(G) \backslash\{\bar{x}\} \text { s.t. } x_{i} \rightarrow \bar{x} \text { and } \nabla G\left(x_{i}\right) \rightarrow \xi\right\}
$$

where $\operatorname{diff}(G)$ denotes the set of differentiability points of $G$ and $\nabla$ is the usual gradient operator. We define the hybrid subdifferential as $\partial^{>} G(\bar{x}):=\operatorname{co} \partial^{*>} G(\bar{x})$. The set $\partial^{*} G(\bar{x})$ is nonempty, closed, in general non convex, and its convex hull coincides with the Clarke subdifferential $\partial^{C} G(\bar{x})$, that is $\partial^{C} G(\bar{x})=\operatorname{co} \partial^{*} G(\bar{x})$. Finally, when $G$ is locally Lipschitz continuous, $\partial^{C} G(\bar{x})=\operatorname{co} \partial G(\bar{x})$.

\section{Optimal Control Problems and a Maximum Principle}

In this section we introduce rigorously the constrained optimization problem over $W^{1,1}$ controls $u$ and its embedding in an extended, or impulsive, problem. Furthermore, we state a Maximum Principle for the extended problem. For simplicity, we will establish all the results for a single state constraint, explaining from time to time with remarks how to adapt these results to the case with $N$ constraints.

Throughout the paper we shall consider the following hypotheses.

(H0) The control set $\mathcal{C} \subseteq \mathbb{R}^{m}$ is a convex cone, the set of ordinary controls $A \subset \mathbb{R}^{q}$ is compact, and the endpoint constraint set $\mathcal{T}_{0} \subseteq \mathbb{R}^{1+n+1+n}$ is closed. 
(H1) The drift function $f \in C^{0}\left(\mathbb{R}^{1+n} \times A ; \mathbb{R}^{n}\right)$ and, for every $a \in A, f(\cdot, a) \in$ $C_{\text {loc }}^{0,1}\left(\mathbb{R}^{1+n} ; \mathbb{R}^{n}\right)$, uniformly w.r.t. $A ; g_{j} \in C_{\text {loc }}^{0,1}\left(\mathbb{R}^{1+n} ; \mathbb{R}^{n}\right)$ for any $j=1, \ldots, m$; $h \in C_{\text {loc }}^{0,1}\left(\mathbb{R}^{1+n} ; \mathbb{R}\right)$.

(H2) The cost function $\Psi \in C^{0,1}\left(\left(\bar{t}_{1}, \bar{x}_{1}, \bar{t}_{2}, \bar{x}_{2}, \bar{v}_{2}\right)+\delta \mathbb{B} ; \mathbb{R}\right)$ for some $\delta>0$, where $\left(\bar{t}_{1}, \bar{x}_{1}, \bar{t}_{2}, \bar{x}_{2}, \bar{v}_{2}\right)$ denotes the endpoints of the optimal trajectory that we will consider in all our results; moreover, for every $\left(t_{1}, x_{1}, t_{2}, x_{2}\right)$, the map $v_{2} \mapsto$ $\Psi\left(t_{1}, x_{1}, t_{2}, x_{2}, v_{2}\right)$ is monotone non-decreasing.

\subsection{The Original Optimal Control Problem}

We set $\left.\left.\mathcal{T}:=\mathcal{T}_{0} \times\right]-\infty, K\right]$ and define the set $\mathcal{U}$ of strict sense controls as

$$
\mathcal{U}:=\left\{\begin{aligned}
\left(t_{1}, t_{2}, u, a\right): t_{1}, t_{2} \in \mathbb{R}, t_{1}<t_{2}, a \in L^{1}\left(\left[t_{1}, t_{2}\right] ; A\right), \\
u \in W^{1,1}\left(\left[t_{1}, t_{2}\right] ; \mathbb{R}^{m}\right), \quad d u(t) / d t \in \mathcal{C} \text { a.e. } t \in\left[t_{1}, t_{2}\right]
\end{aligned}\right\} .
$$

Definition 2.1 (Strict sense processes) Let $\left(t_{1}, t_{2}, u, a\right) \in \mathcal{U}$ be a strict sense control, we call $\left(t_{1}, t_{2}, u, a, x, v\right)$ a strict sense process if the pair $(x, v) \in W^{1,1}\left(\left[t_{1}, t_{2}\right] ; \mathbb{R}^{n+1}\right)$ verifies

$$
\left\{\begin{array}{l}
\frac{d x}{d t}(t)=f(t, x(t), a(t))+\sum_{j=1}^{m} g_{j}(t, x(t)) \frac{d u^{j}}{d t}(t) \\
\frac{d v}{d t}(t)=\left|\frac{d u}{d t}(t)\right|
\end{array} \quad \text { a.e. } t \in\left[t_{1}, t_{2}\right] .\right.
$$

Furthermore, we say that $\left(t_{1}, t_{2}, u, a, x, v\right)$ is feasible if $v\left(t_{1}\right)=0, h(t, x(t)) \leq 0$ for each $t \in\left[t_{1}, t_{2}\right]$ and $\left(t_{1}, x\left(t_{1}\right), t_{2}, x\left(t_{2}\right), v\left(t_{2}\right)\right) \in \mathcal{T}$.

The original optimal control problem is defined as

$$
\left\{\begin{array}{l}
\text { minimize } \Psi\left(t_{1}, x\left(t_{1}\right), t_{2}, x\left(t_{2}\right), v\left(t_{2}\right)\right) \\
\text { over the set of feasible strict sense processes }\left(t_{1}, t_{2}, u, a, x, v\right) .
\end{array}\right.
$$

We consider the following concept of local minimizer.

Definition 2.2 We call a feasible strict sense process $\left(\bar{t}_{1}, \bar{t}_{2}, \bar{u}, \bar{a}, \bar{x}, \bar{v}\right)$ a local strict sense minimizer of $(\mathrm{P})$ if there exists $\delta>0$ such that

$$
\Psi\left(\bar{t}_{1}, \bar{x}\left(\bar{t}_{1}\right), \bar{t}_{2}, \bar{x}\left(\bar{t}_{2}\right), \bar{v}\left(\bar{t}_{2}\right)\right) \leq \Psi\left(t_{1}, x\left(t_{1}\right), t_{2}, x\left(t_{2}\right), v\left(t_{2}\right)\right)
$$

for any feasible $\left(t_{1}, t_{2}, u, a, x, v\right)$ verifying $d_{\infty}\left(\left(\bar{t}_{1}, \bar{t}_{2}, \bar{x}, \bar{v}\right),\left(t_{1}, t_{2}, x, v\right)\right)<\delta$, where $d_{\infty}$ is the distance defined in Eq. 1. If relation (2) is satisfied for all feasible strict sense processes, we say that $\left(\bar{t}_{1}, \bar{t}_{2}, \bar{u}, \bar{a}, \bar{x}, \bar{v}\right)$ is a (global) strict sense minimizer.

Remark 2.1 Arguing similarly to [25], we could consider a more general cost of the form

$$
\Psi\left(t_{1}, x\left(t_{1}\right), t_{2}, x\left(t_{2}\right), v\left(t_{2}\right)\right)+\int_{t_{1}}^{t_{2}} \ell_{0}(t, x(t), a(t))+\ell_{1}(t, x(t),|\dot{u}(t)|) d t,
$$

where $\ell_{0}, \ell_{1}$ are nonnegative and the extended Lagrangian $L$, defined by

$$
L\left(t, x, \omega^{0}, r, a\right):=\ell_{0}(t, x, a) \omega^{0}+\lim _{\rho \rightarrow \omega^{0}} \ell_{1}\left(t, x, \rho^{-1} r\right) \rho,
$$

verifies $L \in C^{0}\left(\mathbb{R} \times \mathbb{R}^{n} \times \mathbb{R}_{\geq 0} \times \mathbb{R}_{\geq 0} \times A ; \mathbb{R}_{\geq 0}\right)$ and, for every $a \in A, L(\cdot, a) \in$ $C_{\text {loc }}^{0,1}\left(\mathbb{R} \times \mathbb{R}^{n} \times \mathbb{R}_{\geq 0} \times \mathbb{R}_{\geq 0} ; \mathbb{R}_{\geq 0}\right)$, uniformly w.r.t. $A$. The results of this article can also 
be applied to the case where dynamics, cost, and constraints depend on the variable $u$. In fact, it is sufficient to add to the control system in (P) the equations $d x^{n+1}(t) / d t=$ $d u^{1}(t) / d t, \ldots, d x^{n+m}(t) / d t=d u^{m}(t) / d t$.

Remark 2.2 As is not difficult to see, given a closed, Hausdorff-Lipschitz continuous multifunction $X:\left[t_{1}, t_{2}\right] \rightsquigarrow \mathbb{R}^{n}$, the function $h(t, x):=d_{X(t)}(x)$ belongs to $C^{0,1}\left(\mathbb{R}^{1+n} ; \mathbb{R}\right)$. Therefore, we could allow implicit time-dependent state constraints of the form $x(t) \in X(t)$ for all $t \in\left[t_{1}, t_{2}\right]$, since one clearly has that $x(t) \in X(t)$ if and only if $h(t, x(t)) \leq 0$ on $\left[t_{1}, t_{2}\right]$.

\subsection{The Extended Optimal Control Problem}

We set

$$
\mathbf{C}:=\left\{\left(\omega^{0}, \omega\right) \in \mathbb{R}_{\geq 0} \times \mathcal{C}: \omega^{0}+|\omega|=1\right\}
$$

and introduce the set of extended sense controls, defined as follows:

$$
\mathcal{W}:=\bigcup_{S>0}\{S\} \times L^{1}([0, S] ; \mathbf{C} \times A) .
$$

Definition 2.3 (Extended sense processes) For any extended sense control $\left(S, \omega^{0}, \omega, \alpha\right) \in$ $\mathcal{W}$, we refer to $\left(S, \omega^{0}, \omega, \alpha, y^{0}, y, \nu\right)$ as an extended sense process if $\left(y^{0}, y, v\right) \in$ $W^{1,1}\left([0, S] ; \mathbb{R}^{1+n+1}\right.$ verifies

$$
\left\{\begin{array}{l}
\frac{d y^{0}}{d s}(s)=\omega^{0}(s) \\
\frac{d y}{d s}(s)=f\left(y^{0}, y, \alpha\right)(s) \omega^{0}(s)+\sum_{i=1}^{m} g_{i}\left(y^{0}, y\right)(s) \omega^{i}(s) \quad \text { a.e. } s \in[0, S] . \\
\frac{d v}{d s}(s)=|\omega(s)|
\end{array}\right.
$$

We say that $\left(S, \omega^{0}, \omega, \alpha, y^{0}, y, v\right)$ is feasible if $v(0)=0, h\left(y^{0}(s), y(s)\right) \leq 0$ for each $s \in[0, S]$ and $\left(y^{0}(0), y(0), y^{0}(S), y(S), v(S)\right) \in \mathcal{T}$.

The set of strict sense processes, say $\Sigma$, can be embedded into the set of extended sense processes, $\Sigma_{e}$, through the following map $\mathcal{I}: \Sigma \rightarrow \Sigma_{e}$, defined as

$$
\mathcal{I}\left(t_{1}, t_{2}, u, a, x, v\right):=\left(S, \omega^{0}, \omega, \alpha, y^{0}, y, v\right),
$$

where, setting $\sigma(t):=t-t_{1}+v(t), S:=\sigma\left(t_{2}\right)$, and $y^{0}:=\sigma^{-1}:[0, S] \rightarrow\left[t_{1}, t_{2}\right]$, we associate to any strict sense process $\left(t_{1}, t_{2}, u, a, x, v\right)$ the extended sense process

$$
\left(S, \omega^{0}, \omega, \alpha, y^{0}, y, v\right):=\left(S, \frac{d y^{0}}{d s},\left(\frac{d u}{d t} \circ y_{0}\right) \cdot \frac{d y^{0}}{d s}, a \circ y^{0}, y^{0}, x \circ y^{0}, v \circ y^{0}\right),
$$

where clearly $\omega^{0}>0$ a.e.. Conversely, if $\left(S, \omega^{0}, \omega, \alpha, y^{0}, y, v\right)$ is an extended sense process with $\omega^{0}>0$ a.e., the absolutely continuous, increasing and surjective inverse $\sigma$ : $\left[t_{1}, t_{2}\right] \rightarrow[0, S]$ of $y^{0}$, allows us to define the strict sense process

$$
\left(t_{1}, t_{2}, u, a, x, v\right):=\left(t_{1}, t_{2}, \int_{\sigma\left(t_{1}\right)}^{\sigma(t)} \omega(s) d s, \alpha \circ \sigma, y \circ \sigma, v \circ \sigma\right) .
$$

\footnotetext{
${ }^{2}$ Since every $L^{1}$-equivalence class contains Borel measurable representatives, we are tacitly assuming that all $L^{1}$-maps we are considering are Borel measurable.
} 
Therefore, $\mathcal{I}$ is injective, ${ }^{3} \mathcal{I}(\Sigma)=\left\{\left(S, \omega^{0}, \omega, \alpha, y^{0}, y, v\right) \in \Sigma_{e}: \omega^{0}>0\right.$ a.e. $\}$, and the extension consists in considering also extended sense processes with $w^{0}$ possibly zero on some non-degenerate intervals. As anticipated in the Introduction, we will sometimes refer to the processes in $\mathcal{I}(\Sigma)$ as embedded strict sense processes.

We define the extended problem as

$$
\left\{\begin{array}{l}
\operatorname{minimize} \Psi\left(y^{0}(0), y(0), y^{0}(S), y(S), v(S)\right) \\
\text { over feasible estended-sense processes }\left(S, \omega^{0}, \omega, \alpha, y^{0}, y, v\right) .
\end{array}\right.
$$

Definition 2.4 A feasible extended sense process $\left(\bar{S}, \bar{\omega}^{0}, \bar{\omega}, \bar{\alpha}, \bar{y}^{0}, \bar{y}, \bar{v}\right)$ is said to be a local minimizer for the extended problem $\left(\mathrm{P}_{e}\right)$ if there exists $\delta>0$ such that

$$
\left.\Psi\left(\bar{y}^{0}(0), \bar{y}(0), \bar{y}^{0}(S), \bar{y}(S), \bar{v}(S)\right)\right) \leq \Psi\left(y^{0}(0), y(0), y^{0}(S), y(S), v(S)\right)
$$

for all feasible extended sense processes $\left(S, \omega^{0}, \omega, \alpha, y^{0}, y, v\right)$ that satisfy $d_{\infty}\left(\left(y^{0}(0)\right.\right.$, $\left.\left.y^{0}(S), y, v\right),\left(\bar{y}^{0}(0), \bar{y}^{0}(\bar{S}), \bar{y}, \bar{v}\right)\right)<\delta$, where $d_{\infty}$ is as in Eq. 1 . If Eq. 8 is satisfied for all feasible extended sense processes, we call $\left(\bar{S}, \bar{\omega}^{0}, \bar{\omega}, \bar{\alpha}, \bar{y}^{0}, \bar{y}, \bar{v}\right)$ a (global) extended sense minimizer.

Remark 2.3 The notion of extended sense local minimizer is the natural extension of the definition of strict sense local minimizer. Indeed, in view of [3, Prop. 2.7], $\left(\bar{t}_{1}, \bar{t}_{2}, \bar{u}, \bar{a}, \bar{x}, \bar{v}\right)$ is a strict sense local minimizer for (P) if and only if $\mathcal{I}\left(\bar{t}_{1}, \bar{t}_{2}, \bar{u}, \bar{a}, \bar{x}, \bar{v}\right)$ is an extended sense local minimizer for $\left(\mathrm{P}_{e}\right)$ among the feasible embedded strict sense processes.

\subsection{A Maximum Principle for the Extended Problem}

Consider the unmaximized Hamiltonian $H$, defined by

$$
H\left(t, x, p_{0}, p, \pi, \omega^{0}, \omega, a\right):=p_{0} \omega^{0}+p \cdot\left(f(t, x, a) \omega^{0}+\sum_{i=1}^{m} g_{i}(t, x) \omega^{i}\right)+\pi|\omega|,
$$

for all $\left(t, x, p_{0}, p, \pi, \omega^{0}, \omega, a\right) \in \mathbb{R}^{1+n+1+n+1} \times \mathbf{C} \times A$.

Theorem 2.1 (PMP) Assume (H0)-(H2). Let $\left(\bar{S}, \bar{\omega}^{0}, \bar{\omega}, \bar{\alpha}, \bar{y}^{0}, \bar{y}, \bar{v}\right)$ be an extended sense local minimizer for $\left(\mathrm{P}_{e}\right)$. Then there exist a path $\left(p_{0}, p\right) \in W^{1,1}\left([0, \bar{S}] ; \mathbb{R} \times \mathbb{R}^{n}\right), \lambda \geq$ $0, \pi \leq 0, \mu \in N B V^{+}([0, \bar{S}] ; \mathbb{R}),\left(m_{0}, m\right):[0, \bar{S}] \rightarrow \mathbb{R}^{1+n}$ Borel measurable and $\mu-$ integrable functions, verifying the following conditions:

(i) (NON-TRIVIALITY)

$$
\left\|p_{0}\right\|_{L^{\infty}}+\|p\|_{L^{\infty}}+\|\mu\|_{T V}+\lambda \neq 0
$$

(ii) (ADJOINT EQUATION) for a.e. $s \in[0, \bar{S}]$,

$$
\left(-\frac{d p_{0}}{d s}(s),-\frac{d p}{d s}(s)\right) \in \operatorname{co} \partial_{t, x} H\left(\bar{y}^{0}(s), \bar{y}(s), q_{0}(s), q(s), \pi, \bar{\omega}^{0}(s), \bar{\omega}(s), \bar{\alpha}(s)\right),
$$

\footnotetext{
${ }^{3}$ Of course, up to translations of $u$.
} 
(iii) (TRANVERSALITY)

$$
\begin{aligned}
\left(p_{0}(0), p(0),-q_{0}(\bar{S}),-q(\bar{S}),-\pi\right) & \in \lambda \partial \Psi\left(\bar{y}^{0}(0), \bar{y}(0), \bar{y}^{0}(\bar{S}), \bar{y}(\bar{S}), \bar{v}(\bar{S})\right) \\
& +N_{\mathcal{T}}\left(\bar{y}^{0}(0), \bar{y}(0), \bar{y}^{0}(\bar{S}), \bar{y}(\bar{S}), \bar{v}(\bar{S})\right) ;
\end{aligned}
$$

(iv) (MAXIMIZATION AND VANISHING OF THE HAMILTONIAN) for a.e. $s \in[0, \bar{S}]$,

$$
\begin{aligned}
H\left(\bar{y}^{0}(s), \bar{y}(s),\right. & \left.q_{0}(s), q(s), \pi, \bar{\omega}^{0}(s), \bar{\omega}(s), \bar{\alpha}(s)\right) \\
& =\max _{\left(\omega^{0}, \omega, a\right) \in \mathbf{C} \times A} H\left(\bar{y}^{0}(s), \bar{y}(s), q_{0}(s), q(s), \pi, \omega^{0}, \omega, a\right)=0 ;
\end{aligned}
$$

(v) $\quad\left(m_{0}, m\right)(s) \in \partial_{t, x}^{>} h\left(\bar{y}^{0}(s), \bar{y}(s)\right) \quad$ h-a.e. $s \in[0, \bar{S}]$;

(vi) $s p t(\mu) \subseteq\left\{s \in[0, \bar{S}]: h\left(\bar{y}^{0}(s), \bar{y}(s)\right)=0\right\}$,

where

$$
\left(q_{0}, q\right)(s):= \begin{cases}\left(p_{0}, p\right)(s)+\int_{[0, s[}\left(m_{0}, m\right)(\tau) \mu(d \tau) & s \in[0, \bar{S}[, \\ \left(p_{0}, p\right)(\bar{S})+\int_{[0, \bar{S}]}\left(m_{0}, m\right)(\tau) \mu(d \tau) & s=\bar{S} .\end{cases}
$$

Furthermore:

(vii) if $\lambda \partial_{v} \Psi\left(\bar{y}^{0}(0), \bar{y}(0), \bar{y}^{0}(\bar{S}), \bar{y}(\bar{S}), \bar{v}(\bar{S})\right)=0$ and $\bar{v}(\bar{S})<K$, then $\pi=0$;

(viii) if $\bar{y}^{0}(0)<\bar{y}^{0}(\bar{S})$, (i) can be strengthened to $\|p\|_{L^{\infty}}+\|\mu\|_{T V}+\lambda \neq 0$.

Proof The extended problem $\left(\mathrm{P}_{e}\right)$ is a conventional optimization problem in the state-space $\left(y^{0}, y, v\right) \in \mathbb{R}^{1+n+1}$, with endpoint constraint $\mathcal{T}$ and state constraint $h\left(y^{0}, y\right) \leq 0$, to which standard 'free end-time' versions of the constrained Maximum Principle are applicable. In particular, the current result can be deduced from [35, Theorem 9.3.1] by means of usual reparameterization techniques (see e.g. [35, Theorem 8.7.1]). Actually, by these arguments it follows the existence of a further multiplier $r \in W^{1,1}([0, \bar{S}] ; \mathbb{R})$ such that

$$
\begin{aligned}
& \frac{d r}{d s}(s)=-H_{s}\left(\bar{y}^{0}(s), \bar{y}(s), q_{0}(s), q(s), \pi, \bar{\omega}^{0}(s), \bar{\omega}(s), \bar{\alpha}(s)\right) \quad \text { a.e. } s \in[0, \bar{S}], \\
& r(\bar{S}) \in-\lambda \Psi_{s}\left(\bar{y}^{0}(0), \bar{y}(0), \bar{y}^{0}(\bar{S}), \bar{y}(\bar{S}), \bar{v}(\bar{S})\right), \\
& \max _{\left(\omega^{0}, \omega, a\right) \in \mathbf{C} \times A} H\left(\bar{y}^{0}(s), \bar{y}(s), q_{0}(s), q(s), \pi, \omega^{0}, \omega, a\right)=r(s) \quad \text { a.e. } s \in[0, \bar{S}],
\end{aligned}
$$

where $H_{s}, \Psi_{s}$ denote the partial derivatives w.r.t. $s$ of $H$ and $\Psi$, respectively. However, since the vector fields $f$ and $\left(g_{j}\right)_{j=1, \ldots, m}$, the cost function $\Psi$, and the constraints do not depend explicitly on the pseudo-time $s$, this yields the constancy of the Hamiltonian with constant equal to 0 in (iv). Finally, the strengthened non-triviality condition (9), which does not involve the multiplier $\pi$ associated to $v$, and the refinements (vii), (viii), can be proved as in [26, Theorem 3.1].

Remark 2.4 (Multiple state constraints) As observed in [35, Section 9], when in problem $\left(\mathrm{P}_{e}\right)$ the single state constraint is replaced with a collection of constraints $h_{i}(t, x) \leq 0, h_{i} \in$ $C_{\text {loc }}^{0,1}\left(\mathbb{R}^{1+n} ; \mathbb{R}\right)$ for $i=1, \ldots, N$, from Theorem 2.1 one can deduce the following corollary: 
given an extended sense local minimizer $\left(\bar{S}, \bar{\omega}^{0}, \bar{\omega}, \bar{\alpha}, \bar{y}^{0}, \bar{y}, \bar{v}\right)$, there exist $\left(p_{0}, p\right) \in W^{1,1}$, $\lambda \geq 0, \pi \leq 0, \mu_{i} \in N B V^{+}([0, \bar{S}] ; \mathbb{R})$ for $i=1, \ldots, N$ and Borel measurable and $\mu_{i^{-}}$ integrable functions $\left(m_{0_{i}}, m_{i}\right)$, such that $\left(m_{0_{i}}, m_{i}\right)(s) \in \partial_{t, x}^{>} h_{i}\left(\bar{y}^{0}(s), \bar{y}(s)\right) \mu_{i}$-a.e. $s \in$ $[0, \bar{S}], \quad s p t\left(\mu_{i}\right) \subseteq\left\{s \in[0, \bar{S}]: h_{i}\left(\bar{y}^{0}(s), \bar{y}(s)\right)=0\right\}$, and conditions (i)-(iv), (vii) and (viii) of Theorem 2.1 are met with $\left(q_{0}, q\right)$ and $\mu$ verifying

$$
\begin{aligned}
& \left(q_{0}, q\right)(s):= \begin{cases}\left(p_{0}, p\right)(s)+\int_{[0, s[} \sum_{i=1}^{N}\left(m_{0_{i}}, m\right)(\tau) \mu_{i}(d \tau) & \text { if } s<\bar{S} \\
\left(p_{0}, p\right)(\bar{S})+\int_{[0, \bar{S}]} \sum_{i=1}^{N}\left(m_{0_{i}}, m\right)(\tau) \mu_{i}(d \tau) & \text { if } s=\bar{S},\end{cases} \\
& \mu(A)=\mu_{1}(A)+\cdots+\mu_{N}(A) \quad \text { for all Borel subsets } A \subseteq[0, \bar{S}] .
\end{aligned}
$$

Definition 2.5 (Normal and abnormal extremal) We say that a feasible extended sense process $\left(\bar{S}, \bar{\omega}^{0}, \bar{\omega}, \bar{\alpha}, \bar{y}^{0}, \bar{y}, \bar{v}\right)$ is an (extended sense) extremal if there exists a set of multipliers $\left(p_{0}, p, \pi, \lambda, \mu\right)$ and functions $m_{0}$ and $m$ which meet the conditions of Theorem 2.1. We will call an extremal normal if all possible choices of multipliers as above have $\lambda>0$, and abnormal when there exists at least one set of such multipliers with $\lambda=0$.

\section{Infimum Gap and Abnormality}

Write $J\left(t_{1}, t_{2}, u, a, x, v\right):=\Psi\left(t_{1}, x\left(t_{1}\right), t_{2}, x\left(t_{2}\right), v\left(t_{2}\right)\right)$ for the cost of a strict sense process $\left(t_{1}, t_{2}, u, a, x, v\right)$ in problem $(\mathrm{P})$, and $J_{e}\left(S, \omega^{0}, \omega, \alpha, y^{0}, y, v\right):=$ $\Psi\left(y^{0}(0), y(0), y^{0}(S), y(S), v(S)\right)$ for the cost of an extended sense process $\left(S, \omega^{0}, \omega, \alpha, y^{0}, y, v\right)$ in problem $\left(\mathrm{P}_{e}\right)$. We also write $\Sigma^{f} \subseteq \Sigma$ and $\Sigma_{e}^{f} \subseteq \Sigma_{e}$ for the subset of feasible strict sense processes and for the subset of feasible extended sense processes, respectively.

Definition 3.1 (Infimum gap) We shall say that there is infimum gap if

$$
\inf _{\Sigma_{e}^{f}} J_{e}\left(S, \omega^{0}, \omega, \alpha, y^{0}, y, v\right)<\inf _{\Sigma^{f}} J\left(t_{1}, t_{2}, u, a, x, v\right) .
$$

Furthermore, if $\left(\bar{S}, \bar{\omega}^{0}, \bar{\omega}, \bar{\alpha}, \bar{y}^{0}, \bar{y}, \bar{v}\right)$ is an extended sense local minimizer, we shall say that there is local infimum gap at $\left(\bar{S}, \bar{\omega}^{0}, \bar{\omega}, \bar{\alpha}, \bar{y}^{0}, \bar{y}, \bar{v}\right)$ if, for some $\delta>0$,

$$
J_{e}\left(\bar{S}, \bar{\omega}^{0}, \bar{\omega}, \bar{\alpha}, \bar{y}^{0}, \bar{y}, \bar{v}\right)<\inf _{B^{\delta}\left(\bar{S}, \bar{\omega}^{0}, \bar{\omega}, \bar{\alpha}, \bar{y}, \bar{y}, \bar{v}\right) \cap \Sigma^{f}} J\left(t_{1}, t_{2}, u, a, x, v\right),
$$

where we have set

$$
\begin{aligned}
& B^{\delta}\left(\bar{S}, \bar{\omega}^{0}, \bar{\omega}, \bar{\alpha}, \bar{y}^{0}, \bar{y}, \bar{v}\right):=\left\{\left(t_{1}, t_{2}, u, a, x, v\right) \in \Sigma:\left(S, \omega^{0}, \omega, \alpha, y^{0}, y, v\right)\right. \\
& \left.\quad:=\mathcal{I}\left(t_{1}, t_{2}, u, a, x, v\right) \text { and } d_{\infty}\left(\left(y^{0}(0), y^{0}(S), y, v\right),\left(\bar{y}^{0}(0), \bar{y}^{0}(\bar{S}), \bar{y}, \bar{v}\right)\right)<\delta\right\} .
\end{aligned}
$$

To prove that, in the presence of a gap, extended sense local minimizers for problem $\left(\mathrm{P}_{e}\right)$ are abnormal extremals, it is convenient to rephrase Definition 3.1 only in terms of extended sense processes. Precisely, using the above notation, by the properties of the map $\mathcal{I}$ (see Eq. 6) it follows that

$$
\begin{aligned}
& \inf _{\Sigma^{f}} J\left(t_{1}, t_{2}, u, a, x, v\right)=\inf _{\mathcal{I}\left(\Sigma^{f}\right)} J_{e}\left(S, \omega^{0}, \omega, \alpha, y^{0}, y, v\right), \\
& \inf _{B^{\delta}\left(\bar{S}, \bar{\omega}^{0}, \bar{\omega}, \bar{\alpha}, \bar{y}^{0}, \bar{y}, \bar{v}\right) \cap \Sigma^{f}} J\left(t_{1}, t_{2}, u, a, x, v\right) \\
& =\inf _{B_{e}^{\delta}\left(\bar{S}, \bar{\omega}^{0}, \bar{\omega}, \bar{\alpha}, \bar{y}^{0}, \bar{y}, \bar{v}\right) \cap \mathcal{I}\left(\Sigma^{f}\right)} J_{e}\left(S, \omega^{0}, \omega, \alpha, y^{0}, y, v\right),
\end{aligned}
$$


where

$$
\begin{aligned}
B_{e}^{\delta}\left(\bar{S}, \bar{\omega}^{0}, \bar{\omega}, \bar{\alpha}, \bar{y}^{0}, \bar{y}, \bar{v}\right):= & \left\{\left(S, \omega^{0}, \omega, \alpha, y^{0}, y, \nu\right) \in \Sigma_{e}:\right. \\
& \left.d_{\infty}\left(\left(y^{0}(0), y^{0}(S), y, \nu\right),\left(\bar{y}^{0}(0), \bar{y}^{0}(\bar{S}), \bar{y}, \bar{v}\right)\right)<\delta\right\} .
\end{aligned}
$$

Even if the set of embedded strict sense processes is dense into the set of extended sense processes with respect to the distance $d_{\infty}$, the infimum gap can actually occur, since all embedded strict sense processes close to a given feasible extended sense process might violate either the endpoint constraints or the state constraint. This leads us to the following definition:

Definition 3.2 (Isolated feasible extended sense process) A feasible extended sense process $\left(S, \omega^{0}, \omega, \alpha, y^{0}, y, v\right)$ is called isolated if, for some $\delta>0$, one has

$$
B_{e}^{\delta}\left(S, \omega^{0}, \omega, \alpha, y^{0}, y, \nu\right) \cap \mathcal{I}\left(\Sigma^{f}\right)=\emptyset .
$$

The following result relates isolated feasible extended sense processes and infimum gap.

Proposition 3.1 Assume (H0)-(H1). Let $\left(\bar{S}, \bar{\omega}^{0}, \bar{\omega}, \bar{\alpha}, \bar{y}^{0}, \bar{y}, \bar{v}\right)$ be an extended sense minimizer [resp., local minimizer] for the extended problem $\left(\mathrm{P}_{e}\right)$ and assume $\Psi \in$ $C^{0}\left(\left(\bar{y}^{0}(0), \bar{y}(0), \bar{y}^{0}(\bar{S}), \bar{y}(\bar{S}), \bar{v}(\bar{S})\right)+\delta \mathbb{B} ; \mathbb{R}\right)$ for some $\delta>0$. If there is infimum gap [resp., local infimum gap at $\left.\left(\bar{S}, \bar{\omega}^{0}, \bar{\omega}, \bar{\alpha}, \bar{y}^{0}, \bar{y}, \bar{v}\right)\right]$, then $\left(\bar{S}, \bar{\omega}^{0}, \bar{\omega}, \bar{\alpha}, \bar{y}{ }^{0}, \bar{y}, \bar{v}\right)$ is an isolated feasible extended sense process.

Proof Suppose by contradiction that $\left(\bar{S}, \bar{\omega}^{0}, \bar{\omega}, \bar{\alpha}, \bar{y}^{0}, \bar{y}, \bar{v}\right)$ is not isolated. Then we can take a sequence $\delta_{j} \downarrow 0$ and, for each $j \in \mathbb{N}$, there exists $\left(S_{j}, \omega_{j}^{0}, \omega_{j}, \alpha_{j}, y_{j}^{0}, y_{j}, v_{j}\right) \in$ $B_{e}^{\delta_{j}}\left(\bar{S}, \bar{\omega}^{0}, \bar{\omega}, \bar{\alpha}, \bar{y}^{0}, \bar{y}, \bar{v}\right) \cap \mathcal{I}\left(\Sigma^{f}\right)$. By the definition of $d_{\infty}$ and the continuity of the cost function $\Psi$, this implies that no infimum gap may occur.

In the following theorem we establish the main result of this section:

Theorem 3.1 Assume (H0)-(H1). Let $\left(\bar{S}, \bar{\omega}^{0}, \bar{\omega}, \bar{\alpha}, \bar{y}^{0}, \bar{y}, \bar{v}\right)$ be an extended sense minimizer [resp., local minimizer] for the extended problem $\left(\mathrm{P}_{e}\right)$ and assume $\Psi \in$ $C^{0}\left(\left(\bar{y}^{0}(0), \bar{y}(0), \bar{y}^{0}(\bar{S}), \bar{y}(\bar{S}), \bar{v}(\bar{S})\right)+\delta \mathbb{B} ; \mathbb{R}\right)$ for some $\delta>0$. If there is infimum gap [resp., local infimum gap at $\left.\left(\bar{S}, \bar{\omega}^{0}, \bar{\omega}, \bar{\alpha}, \bar{y}^{0}, \bar{y}, \bar{v}\right)\right]$, then $\left(\bar{S}, \bar{\omega}^{0}, \bar{\omega}, \bar{\alpha}, \bar{y}^{0}, \bar{y}, \bar{v}\right)$ is an abnormal extremal.

Thanks to Proposition 3.1, Theorem 3.1 is a straightforward consequence of the following result, which extends [26, Th. 4.4] to the case with state constraints, an additional ordinary control in the drift, and nonsmooth data.

Theorem 3.2 Assume (H0)-(H1). If $\left(\bar{S}, \bar{\omega}^{0}, \bar{\omega}, \bar{\alpha}, \bar{y}^{0}, \bar{y}, \bar{v}\right)$ is an isolated feasible extended sense process, then it is an abnormal extremal.

Proof Since the proof involves only space-time trajectories $\left(y^{0}, y\right)$ which are close to the reference space-time trajectory $\left(\bar{y}^{0}, \bar{y}\right)$ and the controls assume values in a compact set, using standard truncation and mollification arguments we can assume that there exists some $L>0$ such that the functions $f, g_{1}, \ldots, g_{m}$, and $h$ are $L$-Lipschitz continuous and bounded by $L$. The proof is divided into several steps in which successive sequences of optimization 
problems are introduced that have as eligible controls only embedded strict controls, and costs that measure how much a process violates the constraints. Using the Ekeland Principle, minimizers are then built for these problems, which converge to the initial isolated process. Furthermore, applying the PMP to these approximate problems with reference to the above mentioned minimizers, we obtain in the limit a set of multipliers with $\lambda=0$ for problem $\left(\mathrm{P}_{e}\right)$, with reference to the isolated process $\left(\bar{S}, \bar{\omega}^{0}, \bar{\omega}, \bar{\alpha}, \bar{y}^{0}, \bar{y}, \bar{v}\right)$.

Step 1 . Define the function $\Phi: \mathbb{R}^{1+n+1+n+1} \rightarrow \mathbb{R}$, given by

$$
\Phi\left(t_{1}, x_{1}, t_{2}, x_{2}, v_{2}\right):=d_{\mathcal{T}_{0}}\left(t_{1}, x_{1}, t_{2}, x_{2}\right) \vee\left[\left(v_{2}-K\right) \vee 0\right]
$$

and for any $\left(y^{0}, y, v\right) \in W^{1,1}\left([0, \bar{S}] ; \mathbb{R}^{1+n+1}\right)$, introduce the payoff

$$
\mathcal{J}\left(y^{0}, y, v\right):=\Phi\left(y^{0}(0), y(0), y^{0}(\bar{S}), y(\bar{S}), v(\bar{S})\right) \vee \max _{s \in[0, \bar{S}]} h\left(y^{0}(s), y(s)\right) .
$$

Fixed a sequence $\left(\varepsilon_{i}\right)_{i}$ such that $\varepsilon_{i} \downarrow 0$, for each $i \in \mathbb{N}$ we consider the fixed end-time optimal control problem:

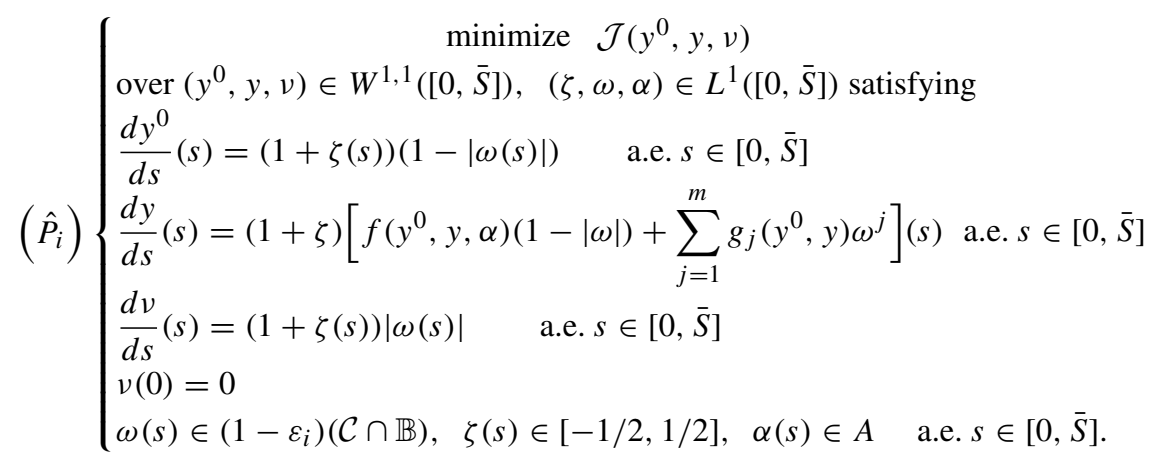

We will call an element $\left(\zeta, \omega, \alpha, y^{0}, y, v\right)$ satisfying the constraints in $\left(\hat{P}_{i}\right)$ a feasible process for problem $\left(\hat{P}_{i}\right)$. For every $i \in \mathbb{N}$, let $\left(\bar{S}, \hat{\omega}_{i}^{0}, \hat{\omega}_{i}, \hat{\alpha}_{i}, \hat{y}_{i}^{0}, \hat{y}_{i}, \hat{v}_{i}\right)$ be the extended sense process in which $\left(\hat{y}_{i}^{0}, \hat{y}_{i}, \hat{v}_{i}\right)(0)=\left(\bar{y}^{0}, \bar{y}, \bar{v}\right)(0)$ and

$$
\left(\hat{\omega}_{i}^{0}, \hat{\omega}_{i}, \hat{\alpha}_{i}\right)(s):= \begin{cases}\left(\varepsilon_{i},\left(1-\varepsilon_{i}\right) \frac{\bar{\omega}(s)}{|\bar{\omega}(s)|}, \bar{\alpha}(s)\right) & \text { if } \bar{\omega}^{0}(s)<\varepsilon_{i} \\ \left(\bar{\omega}^{0}(s), \bar{\omega}(s), \bar{\alpha}(s)\right) & \text { if } \bar{\omega}^{0}(s) \geq \varepsilon_{i} .\end{cases}
$$

Notice that $\left(\bar{S}, \hat{\omega}_{i}^{0}, \hat{\omega}_{i}, \hat{\alpha}_{i}, \hat{y}_{i}^{0}, \hat{y}_{i}, \hat{v}_{i}\right)$ is an embedded strict sense process for the extended problem $\left(\mathrm{P}_{e}\right)$, since $\hat{\omega}_{i}^{0}(s) \geq \varepsilon_{i}>0$ for a.e. $s \in[0, \bar{S}]$. Moreover, $\left(\hat{\zeta}_{i}, \hat{\omega}_{i}, \hat{\alpha}_{i}, \hat{y}_{i}^{0}, \hat{y}_{i}, \hat{v}_{i}\right)$ with $\hat{\zeta}_{i} \equiv 0$ is a feasible process for $\left(\hat{P}_{i}\right)$, since $\hat{\omega}_{i}(s) \in\left(1-\varepsilon_{i}\right)(\mathcal{C} \cap \mathbb{B})$ a.e. $s \in[0, \bar{S}]$. Furthermore,

$$
\hat{\alpha}_{i} \equiv \bar{\alpha}, \quad\left\|\left(\hat{\omega}_{i}^{0}, \hat{\omega}_{i}\right)-\left(\bar{\omega}^{0}, \bar{\omega}\right)\right\|_{L^{\infty}([0, \bar{S}])} \rightarrow 0 \quad \text { as } i \rightarrow \infty,
$$

therefore, by the continuity of the input-output map $(\zeta, \omega, \alpha) \mapsto\left(y^{0}, y, v\right)$, we have:

$$
\left\|\left(\hat{y}_{i}^{0}, \hat{y}_{i}, \hat{v}_{i}\right)-\left(\bar{y}^{0}, \bar{y}, \bar{v}\right)\right\|_{L^{\infty}([0, \bar{S}])} \rightarrow 0 \quad \text { as } i \rightarrow \infty .
$$

Since $\mathcal{J}$ is nonnegative and vanishes at $\left(\bar{S}, \bar{\omega}^{0}, \bar{\omega}, \bar{\alpha}, \bar{y}^{0}, \bar{y}, \bar{v}\right)$, by the $L$-Lipschitz continuity of $h$ and the 1-Lipschitz continuity of $d_{\mathcal{T}_{0}}(\cdot)$, Eq. 15 implies that there exist a sequence $\rho_{i} \downarrow 0$ such that, for every $i \in \mathbb{N},\left(0, \hat{\omega}_{i}, \hat{\alpha}_{i}, \hat{y}_{i}^{0}, \hat{y}_{i}, \hat{v}_{i}\right)$ has cost not greater than $\rho_{i}^{2}$, namely is a $\rho_{i}^{2}$-minimizer for the problem $\left(\hat{P}_{i}\right)$. 
Step 2. If we endow the set of feasible processes for problem $\left(\hat{P}_{i}\right)$, say $\hat{\Gamma}_{i}$, with the distance

$$
\begin{aligned}
\hat{d}\left(\left(\zeta, \omega, \alpha, y^{0}, y, v\right),\left(\tilde{\zeta}, \tilde{\omega}, \tilde{\alpha}, \tilde{y}^{0}, \tilde{y}, \tilde{v}\right)\right):=\left|\left(y^{0}, y\right)(0)-\left(\tilde{y}^{0}, \tilde{y}\right)(0)\right| \\
+\|\omega-\tilde{\omega}\|_{L^{1}([0, \bar{S}])}+\ell\{s \in[0, \bar{S}]:(\zeta(s), \alpha(s)) \neq(\tilde{\zeta}(s), \tilde{\alpha}(s))\}
\end{aligned}
$$

for every pair $\left(\zeta, \omega, \alpha, y^{0}, y, v\right),\left(\tilde{\zeta}, \tilde{\omega}, \tilde{\alpha}, \tilde{y}^{0}, \tilde{y}, \tilde{v}\right) \in \hat{\Gamma}_{i},\left(\hat{P}_{i}\right)$ can be seen as aptimization problem with continuous cost over the complete metric space $\left(\hat{\Gamma}_{i}, \hat{d}\right)$. Hence, by Ekeland's Principle, if we introduce the function

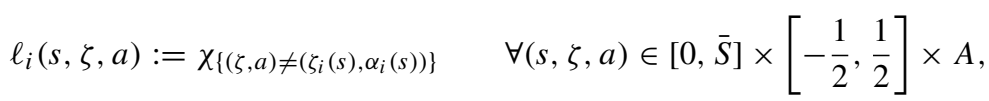

for any $i \in \mathbb{N}$ there is a feasible process $\left(\zeta_{i}, \omega_{i}, \alpha_{i}, y_{i}^{0}, y_{i}, v_{i}\right)$ for $\left(\hat{P}_{i}\right)$ which is a minimizer of

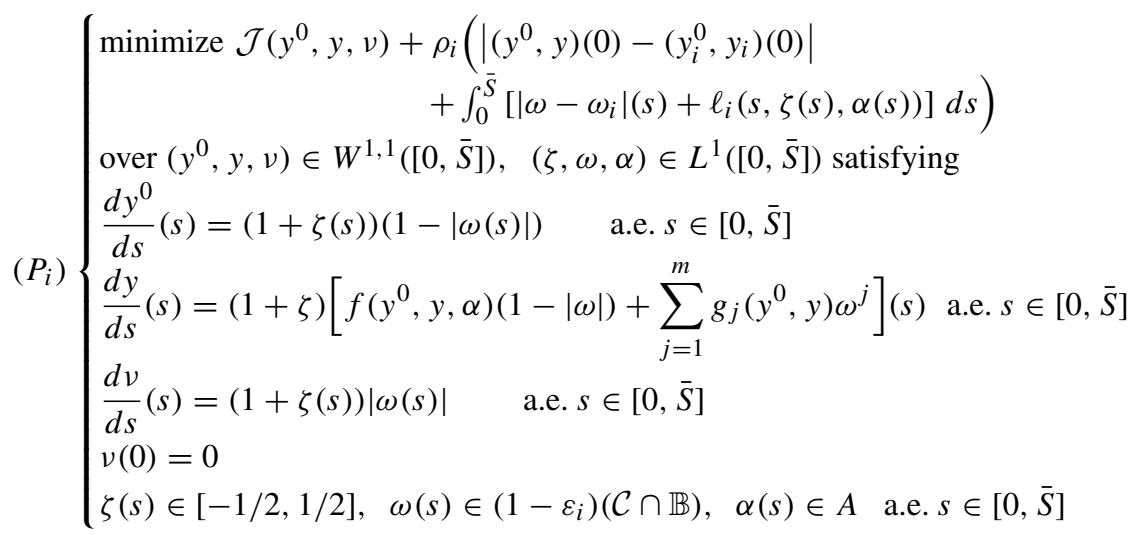

and verifies

$$
\left|\left(\hat{y}_{i}^{0}, \hat{y}_{i}\right)(0)-\left(y_{i}^{0}, y_{i}\right)(0)\right|+\int_{0}^{\bar{s}}\left[\left|\hat{\omega}_{i}-\omega_{i}\right|(s)+\ell_{i}(s, 0, \bar{\alpha}(s))\right] d s \leq \rho_{i} \rightarrow 0 .
$$

Thus, by Eqs. 14 and 16 it follows that, as $i \rightarrow+\infty$,

$$
\begin{gathered}
\left\|\left(y_{i}^{0}, y_{i}, v_{i}\right)-\left(\bar{y}^{0}, \bar{y}, \bar{v}\right)\right\|_{L^{\infty}([0, \bar{S}])} \rightarrow 0, \quad\left\|\omega_{i}-\bar{\omega}\right\|_{L^{1}([0, \bar{S}])} \rightarrow 0 \\
\ell\left\{s \in[0, \bar{S}]:\left(\zeta_{i}(s), \alpha_{i}(s)\right) \neq(0, \bar{\alpha}(s))\right\} \rightarrow 0
\end{gathered}
$$

so that, eventually passing to a subsequence, $\left(\omega_{i}\right)_{i}$ converges to $\bar{\omega}$ almost everywhere.

Let us now show that, through suitable reparameterization techniques, the sequence of minimizing processes $\left(\zeta_{i}, \omega_{i}, \alpha_{i}, y_{i}^{0}, y_{i}, \nu_{i}\right)$ can be associated to a sequence of embedded strict processes converging to the original isolated process $\left(\bar{S}, \bar{\omega}^{0}, \bar{\omega}, \bar{\alpha}, \bar{y}^{0}, \bar{y}, \bar{v}\right)$.

Precisely, for each $i \in \mathbb{N}$, let us consider the surjective, bi-Lipschitz continuous, and strictly increasing function $\sigma_{i}:[0, \bar{S}] \rightarrow\left[0, \tilde{S}_{i}\right]$, given by

$$
\sigma_{i}(s):=\int_{0}^{s}\left(1+\zeta_{i}(r)\right) \mathrm{d} r, \quad \tilde{S}_{i}:=\sigma_{i}(\bar{S}) .
$$

Using as reparameterization the inverse function $\sigma_{i}^{-1}:\left[0, \tilde{S}_{i}\right] \rightarrow[0, \bar{S}]$, we derive that the corresponding process $\left(\tilde{S}_{i}, \tilde{\omega}_{i}^{0}, \tilde{\omega}_{i}, \tilde{\alpha}_{i}, \tilde{y}_{i}^{0}, \tilde{y}_{i}, \tilde{v}_{i}\right)$, where

$$
\left(\tilde{y}_{i}^{0}, \tilde{y}_{i}, \tilde{v}_{i}\right):=\left(y_{i}^{0}, y_{i}, v_{i},\right) \circ \sigma_{i}^{-1}, \quad\left(\tilde{\omega}_{i}^{0}, \tilde{\omega}_{i}, \tilde{\alpha}_{i}\right):=\left(1-\left|\omega_{i}\right|, \omega_{i}, \alpha_{i}\right) \circ \sigma_{i}^{-1},
$$


is an embedded strict sense process for problem $\left(\mathrm{P}_{e}\right)$. In particular, we have

$$
\begin{aligned}
& \tilde{\omega}_{i}^{0}(s)=1-\left|\tilde{\omega}_{i}(s)\right|, \quad \tilde{\omega}_{i}(s) \in\left(1-\epsilon_{i}\right)\left(\mathcal{C} \cap \mathbb{B}_{m}\right) \text { a.e. } s \in\left[0, \tilde{S}_{i}\right], \\
& \left(\tilde{y}_{i}^{0}(0), \tilde{y}_{i}(0), \tilde{y}_{i}^{0}\left(\tilde{S}_{i}\right), \tilde{y}_{i}\left(\tilde{S}_{i}\right), \tilde{v}_{i}\left(\tilde{S}_{i}\right)\right)=\left(y_{i}^{0}(0), y_{i}(0), y_{i}^{0}(\bar{S}), y_{i}(\bar{S}), v_{i}(\bar{S})\right) .
\end{aligned}
$$

Hence, we deduce from Eq. 17 that, for $i$ sufficiently large,

$$
d_{\infty}\left(\left(\tilde{y}_{i}^{0}(0), \tilde{y}_{i}^{0}\left(\tilde{S}_{i}\right), \tilde{y}_{i}, \tilde{v}_{i}\right),\left(\bar{y}^{0}(0), \bar{y}^{0}(\bar{S}), \bar{y}, \bar{v}\right)\right)<\delta,
$$

where $\delta>0$ is the constant appearing in Definition 3.2, with reference to the isolated feasible extended sense process $\left(\bar{S}, \bar{\omega}^{0}, \bar{\omega}, \bar{\alpha}, \bar{y}^{0}, \bar{y}, \bar{v}\right)$. As a consequence, for all $i$ large enough, $\left(\tilde{S}_{i}, \tilde{\omega}_{i}^{0}, \tilde{\omega}_{i}, \tilde{\alpha}_{i}, \tilde{y}_{i}^{0}, \tilde{y}_{i}, \tilde{v}_{i}\right)$ cannot be a feasible embedded strict sense process, namely, it must violate either the endpoint constraints or the state constraint. By Eqs. 19 and 20 this implies that $\mathcal{J}\left(y_{i}^{0}, y_{i}, v_{i}\right)>0$, namely, at least one of the following three inequalities holds true:

$$
d_{\mathcal{T}_{0}}\left(y_{i}^{0}(0), y_{i}(0), y_{i}^{0}(\bar{S}), y_{i}(\bar{S})\right)>0, \quad v_{i}(\bar{S})>K, \quad \max _{s \in[0, \bar{S}]} h\left(y_{i}^{0}(s), y_{i}(s)\right)>0 .
$$

In the following, as is clearly not restrictive, we will always assume that the properties valid from a certain index onwards, apply to each index $i \in \mathbb{N}$.

Step 3. For each $i \in \mathbb{N}$, define $c_{i}:=\max _{s \in[0, \bar{S}]} h\left(y_{i}^{0}(s), y_{i}(s)\right)$ and set

$$
\tilde{h}(t, x, c):=h(t, x)-c \quad \forall(t, x, c) \in \mathbb{R}^{1+n+1} .
$$

The process $\left(\zeta_{i}, \omega_{i}, \alpha_{i}, y_{i}^{0}, y_{i}, v_{i}, c_{i}\right)$ turns out to be a minimizer for

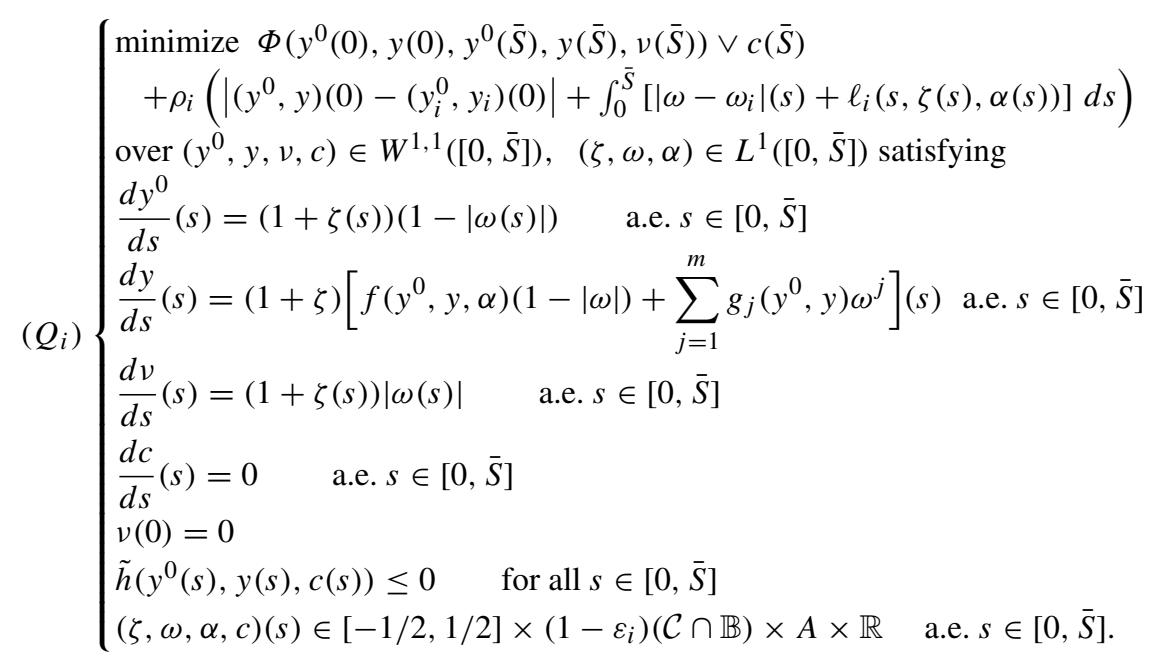

Our aim is now to apply the Pontryagin Maximum Principle to problem $\left(Q_{i}\right)$ with reference to the minimizer $\left(\zeta_{i}, \omega_{i}, \alpha_{i}, y_{i}^{0}, y_{i}, v_{i}, c_{i}\right)$. Preliminarily, let us observe that, passing eventually to a subsequence, we may assume that either $c_{i}>0$ for each $i \in \mathbb{N}$ or $c_{i} \leq 0$ for each $i \in \mathbb{N}$.

Assume first that $c_{i}>0$ for each $i \in \mathbb{N}$. Fix $i \in \mathbb{N}$ and set $\omega_{i}^{0}:=1-\left|\omega_{i}\right|$. In the Maximum Principle, several generalized subdifferentials are involved which it is convenient to make as explicit as possible. First of all, the condition ' $h\left(y_{i}^{0}(s), y_{i}(s)\right)-c_{i}>0$ ' implies ' $h\left(y_{i}^{0}(s), y_{i}(s)\right)>c_{i}>0$ ', so that $\partial_{t, x, c}^{>} \tilde{h}\left(y_{i}^{0}(s), y_{i}(s), c_{i}\right)=\partial_{t, x}^{>} h\left(y_{i}^{0}(s), y_{i}(s)\right) \times\{-1\}$. Furthermore, by the 'max rule' of subdifferential calculus (see [35, Th. 5.5.2]), the properties of the subdifferential of the distance function (see [35, Lemma 4.8.3]), and Eq. 22, 
we have that $\left(\gamma_{0_{i}}^{1}, \gamma_{i}^{1}, \gamma_{0_{i}}^{2}, \gamma_{i}^{2}, \gamma_{v_{i}}^{2}, \gamma_{c_{i}}^{2}\right) \in \partial\left(\Phi\left(y^{0}(0), y(0), y^{0}(\bar{S}), y(\bar{S}), \nu(\bar{S})\right) \vee c(\bar{S})\right)$ implies that there are some $\sigma_{i}^{1}, \sigma_{i}^{2}, \sigma_{i}^{3} \geq 0$ with $\sum_{k=1}^{3} \sigma_{i}^{k}=1$, such that

$$
\begin{aligned}
& \left(\gamma_{0_{i}}^{1}, \gamma_{i}^{1}, \gamma_{0_{i}}^{2}, \gamma_{i}^{2}\right) \in \sigma_{i}^{1}\left(\partial d_{\mathcal{T}_{0}}\left(y_{i}^{0}(0), y_{i}(0), y_{i}^{0}(\bar{S}), y_{i}(\bar{S})\right) \cap \partial \mathbb{B}_{1+n+1+n}\right), \\
& \gamma_{v_{i}}^{2}=\sigma_{i}^{2} \quad\left(\text { since } \partial\left(\left(v_{i}(\bar{S})-K\right) \vee 0\right)=1 \text { when } v_{i}(\bar{S})>K\right), \quad \gamma_{c_{i}}^{2}=\sigma_{i}^{3},
\end{aligned}
$$

and $\sigma_{i}^{k}=0$ when the maximum in $\Phi\left(y_{i}^{0}(0), y_{i}(0), y_{i}^{0}(\bar{S}), y_{i}(\bar{S}), v_{i}(\bar{S})\right) \vee c_{i}(\bar{S})$ is strictly greater than the $k$-th term in the maximization. Thus, the Maximum Principle in [35, Th. 9.3.1] yields the existence of some multipliers $\left(p_{0_{i}}, p_{i}, \pi_{i}, r_{i}\right) \in W^{1,1}\left([0, \bar{S}] ; \mathbb{R}^{1+n+1+1}\right)$ associated with $\left(y_{i}^{0}, y_{i}, v_{i}, c_{i}\right), \mu_{i} \in N B V^{+}([0, \bar{S}] ; \mathbb{R}), \lambda_{i} \geq 0, \sigma_{i}^{1}, \sigma_{i}^{2}, \sigma_{i}^{3} \geq 0$ with $\sum_{k=1}^{3} \sigma_{i}^{k}=1$, and Borel-measurable, $\mu_{i}$-integrable functions $\left(m_{0_{i}}, m_{i}\right):[0, \bar{S}] \rightarrow \mathbb{R}^{1+n}$, such that:

(i) $^{\prime} \quad\left\|p_{0_{i}}\right\|_{L^{\infty}}+\left\|p_{i}\right\|_{L^{\infty}}+\left\|\mu_{i}\right\|_{T V}+\lambda_{i}+\left\|r_{i}\right\|_{L^{\infty}}+\left\|\pi_{i}\right\|_{L^{\infty}}=1 ;$

(ii) $^{\prime} \quad\left(-\frac{d p_{0_{i}}}{d s}(s),-\frac{d p_{i}}{d s}(s)\right) \in \operatorname{co} \partial_{t, x}\left\{\left[q_{i}(s) \cdot\left(f\left(\left(y_{i}^{0}, y_{i}, \alpha_{i}\right)(s)\right) \omega_{i}^{0}(s)\right.\right.\right.$

$$
\left.\left.\left.+\sum_{j=1}^{m} g_{j}\left(\left(y_{i}^{0}, y_{i}\right)(s)\right) \omega_{i}^{j}(s)\right)\right]\left(1+\zeta_{i}(s)\right)\right\}
$$

and $d \pi_{i}(s) / d s=d r_{i}(s) / d s=0$ for a.e. $s \in[0, \bar{S}]$;

(iii) $^{\prime} \quad\left(p_{0_{i}}(0), p_{i}(0),-q_{0_{i}}(\bar{S}),-q_{i}(\bar{S})\right) \in \lambda_{i} \rho_{i} \mathbb{B}_{1+n} \times\left\{0_{1+n}\right\}$

$+\lambda_{i} \sigma_{i}^{1}\left(\partial d_{\mathcal{T}_{0}}\left(y_{i}^{0}(0), y_{i}(0), y_{i}^{0}(\bar{S}), y_{i}(\bar{S})\right) \cap \partial \mathbb{B}_{1+n+1+n}\right)$,

$-\pi_{i}=\lambda_{i} \sigma_{i}^{2}, \quad r_{i}(0)=0, \quad-r_{i}(\bar{S})+\int_{[0, \bar{S}]} \mu_{i}(d s)=\lambda_{i} \sigma_{i}^{3}$;

(iv) $^{\prime} \quad \int_{0}^{\bar{S}}\left\{\left[q_{i} \cdot\left(f\left(y_{i}^{0}, y_{i}, \alpha_{i}\right) \omega_{i}^{0}+\sum_{j=1}^{m} g_{j}\left(y_{i}^{0}, y_{i}\right) \omega_{i}^{j}\right)+q_{0_{i}} \omega_{i}^{0}+\pi_{i}\left|\omega_{i}\right|\right]\left(1+\zeta_{i}\right)\right\} \mathrm{d} s$

$\geq \int_{0}^{\bar{S}}\left\{\left[q_{i} \cdot\left(f\left(y_{i}^{0}, y_{i}, \alpha\right) \omega^{0}+\sum_{j=1}^{m} g_{j}\left(y_{i}^{0}, y_{i}\right) \omega^{j}\right)+q_{0_{i}} \omega^{0}+\pi_{i}|\omega|\right](1+\zeta)\right.$

$\left.-\lambda_{i} \rho_{i}\left[\left|\omega-\omega_{i}\right|+\ell_{i}(s, \zeta(s), \alpha(s))\right]\right\} \mathrm{d} s$

for all measurable selectors $(\zeta, \omega, \alpha)$ of $\left[-\frac{1}{2}, \frac{1}{2}\right] \times\left(1-\varepsilon_{i}\right)\left(\mathcal{C} \cap \mathbb{B}_{m}\right) \times A$;

$(\mathrm{v})^{\prime} \quad\left(m_{0_{i}}, m_{i}\right)(s) \in \partial_{t, x}^{>} h\left(y_{i}^{0}(s), y_{i}(s)\right) \quad \mu_{i}$-a.e. $s \in[0, \bar{S}]$,

(vi) $\quad s p t\left(\mu_{i}\right) \subseteq\left\{s: h\left(y_{i}^{0}(s), y_{i}(s)\right)-c_{i}=0\right\}$,

where

$$
\left(q_{0_{i}}, q_{i}\right)(s):= \begin{cases}\left(p_{0_{i}}, p_{i}\right)(s)+\int_{[0, s[}\left(m_{0_{i}}, m_{i}\right)(\tau) \mu_{i}(d \tau) & s \in[0, \bar{S}[, \\ \left(p_{0_{i}}, p_{i}\right)(\bar{S})+\int_{[0, \bar{S}]}\left(m_{0_{i}}, m_{i}\right)(\tau) \mu_{i}(d \tau) & s=\bar{S} .\end{cases}
$$

Observe that, for each $i \in \mathbb{N}$, by (ii) ${ }^{\prime}$ and (iii) ${ }^{\prime}$ we have

$$
r_{i} \equiv 0, \quad\left\|\mu_{i}\right\|_{T V}=\int_{[0, \bar{S}]} \mu_{i}(d s)=\lambda_{i} \sigma_{i}^{3}, \quad\left|\pi_{i}\right|=\lambda_{i} \sigma_{i}^{2}
$$

furthermore, $\left\|\left(m_{0_{i}}, m_{i}\right)\right\|_{L^{\infty}} \leq L$ by $(\mathrm{v})^{\prime}$ and the $L$-Lipschitz continuity of $h$. Then by (iii) ${ }^{\prime}$ and Eq. 23, we get

$$
\begin{aligned}
\lambda_{i}\left(1-\sigma_{i}^{3}\right)-\lambda_{i} \rho_{i} & \leq\left|\left(p_{0_{i}}(0), p_{i}(0),-q_{0_{i}}(\bar{S}),-q_{i}(\bar{S})\right)\right|+\left|\pi_{i}\right| \\
& \leq 2\left\|p_{0_{i}}\right\|_{L^{\infty}}+2\left\|p_{i}\right\|_{L^{\infty}}+2 L\left\|\mu_{i}\right\|_{T V}+\left|\pi_{i}\right| .
\end{aligned}
$$


By this estimate, Eq. 23, the non-triviality condition (i) ${ }^{\prime}$, and using the facts that $\rho_{i} \leq \frac{1}{2}$ for $i$ sufficiently large and $\lambda_{i} \in[0,1]$, for such $i$ we get

$$
\begin{aligned}
3\left\|p_{0_{i}}\right\|_{L^{\infty}} & +3\left\|p_{i}\right\|_{L^{\infty}}+(2 L+2)\left\|\mu_{i}\right\|_{T V}+3\left|\pi_{i}\right| \\
& \geq \lambda_{i}\left(1-\sigma_{i}^{3}\right)-\lambda_{i} \rho_{i}+1-\lambda_{i}+\lambda_{i} \sigma_{i}^{2}+\lambda_{i} \sigma_{i}^{3}=1+\lambda_{i} \sigma_{i}^{2}-\lambda_{i} \rho_{i} \geq \frac{1}{2} .
\end{aligned}
$$

Hence, scaling the multipliers, we obtain

$$
\left\|p_{0_{i}}\right\|_{L^{\infty}}+\left\|p_{i}\right\|_{L^{\infty}}+\left\|\mu_{i}\right\|_{T V}+\left|\pi_{i}\right|=1, \quad \lambda_{i} \leq \tilde{L}:=6 \vee 4(1+L) .
$$

Suppose now $c_{i} \leq 0$ for each $i \in \mathbb{N}$. In this case, by Eq. 22, either $v_{i}(\bar{S})>K$ or $d_{\mathcal{T}_{0}}\left(y_{i}^{0}(0), y_{i}(0), \overline{y_{i}^{0}}(\bar{S}), y_{i}(\bar{S})\right)>0$. Thus, for $\varepsilon>0$ suitably small, the process $\left(\zeta_{i}, \omega_{i}, \alpha_{i}, y_{i}^{0}, y_{i}, v_{i}, \hat{c}_{i}\right)$ with $\hat{c}_{i}:=c_{i}+\varepsilon$ is still a minimizer for problem $\left(Q_{i}\right)$ and, in addition, it verifies $h\left(y_{i}^{0}(s), y_{i}(s)\right)-\hat{c}_{i}<0$ for all $s \in[0, \bar{S}]$ (namely, the state constraint is inactive on $[0, \bar{S}])$. Hence, by applying the Maximum Principle for problem $\left(Q_{i}\right)$ with reference to this minimizer we deduce the existence of multipliers $\left(p_{0_{i}}, p_{i}, \pi_{i}, r_{i}\right) \in$ $W^{1,1}\left([0, \bar{S}] ; \mathbb{R}^{1+n+1+1}\right)$, which satisfy conditions (i)'-(vi)' with $\mu_{i}=0, \sigma_{i}^{3}=0, \pi_{i} \leq 0$, and $\lambda_{i}>0$. In this case, by considering again $i$ sufficiently large to have $\rho_{i} \leq \frac{1}{2}$, from (iii) we get

$$
\lambda_{i}\left(1-\rho_{i}\right) \leq\left|\left(p_{0_{i}}(0), p_{i}(0),-q_{0_{i}}(\bar{S}),-q_{i}(\bar{S})\right)\right|+\left|\pi_{i}\right| \leq 2\left\|p_{0_{i}}\right\|_{L^{\infty}}+2\left\|p_{i}\right\|_{L^{\infty}}+\left|\pi_{i}\right|,
$$

and, scaling the multipliers appropriately after summing (i)', we finally obtain

$$
\left\|p_{0_{i}}\right\|_{L^{\infty}}+\left\|p_{i}\right\|_{L^{\infty}}+\left|\pi_{i}\right|=1, \quad \lambda_{i} \leq 6 \quad(\leq \tilde{L}) .
$$

Step 4. From the previous step, we arrive at the following properties (for either the case where $c_{i}>0$ for each $i \in \mathbb{N}$ or the case where $c_{i} \leq 0$ for each $i \in \mathbb{N}$ ): for any $i \in \mathbb{N}$, there exist $\left(p_{0_{i}}, p_{i}\right) \in W^{1,1}\left([0, \bar{S}] ; \mathbb{R}^{1+n}\right), \pi_{i} \leq 0, \mu_{i} \in N B V^{+}([0, \bar{S}] ; \mathbb{R})$ and Borelmeasurable, $\mu_{i}$-integrable functions $\left(m_{0_{i}}, m_{i}\right):[0, \bar{S}] \rightarrow \mathbb{R}^{1+n}$, such that:

(i) $\left\|p_{0_{i}}\right\|_{L^{\infty}}+\left\|p_{i}\right\|_{L^{\infty}}+\left\|\mu_{i}\right\|_{T V}+\left|\pi_{i}\right|=1$,

(ii) $\quad\left(-\frac{d p_{0_{i}}}{d s}(s),-\frac{d p_{i}}{d s}(s)\right) \in \operatorname{co} \partial_{t, x}\left\{\left[q_{i}(s) \cdot\left(f\left(y_{i}^{0}(s), y_{i}(s), \alpha_{i}(s)\right) \omega_{i}^{0}(s)\right.\right.\right.$

$\left.\left.\left.+\sum_{j=1}^{m} g_{j}\left(y_{i}^{0}(s), y_{i}(s)\right) \omega_{i}^{j}(s)\right)\right]\left(1+\zeta_{i}(s)\right)\right\}$ for a.e. $s \in[0, \bar{S}]$;

(iii) $\quad\left(p_{0_{i}}(0), p_{i}(0),-q_{0_{i}}(\bar{S}),-q_{i}(\bar{S}),-\pi_{i}\right)$ $\in[0, \tilde{L}] \cdot \partial \Phi\left(y_{i}^{0}(0), y_{i}(0), y_{i}^{0}(\bar{S}), y_{i}(\bar{S}), v_{i}(\bar{S})\right)+\tilde{L} \rho_{i} \mathbb{B}_{1+n} \times\left\{0_{1+n}\right\} \times\{0\} ;$

$\int_{0}^{\bar{S}}\left\{\left[q_{i} \cdot\left(f\left(y_{i}^{0}, y_{i}, \alpha_{i}\right) \omega_{i}^{0}+\sum_{j=1}^{m} g_{j}\left(y_{i}^{0}, y_{i}\right) \omega_{i}^{j}\right)+q_{0_{i}} \omega_{i}^{0}+\pi_{i}\left|\omega_{i}\right|\right]\left(1+\zeta_{i}\right)\right\} \mathrm{d} s$

$\geq \int_{0}^{\bar{S}}\left\{\left[q_{i} \cdot\left(f\left(y_{i}^{0}, y_{i}, \alpha\right) \omega^{0}+\sum_{j=1}^{m} g_{j}\left(y_{i}^{0}, y_{i}\right) \omega^{j}\right)\right.\right.$

$\left.\left.+q_{0_{i}} \omega^{0}+\pi_{i}|\omega|\right](1+\zeta)-3 \tilde{L} \rho_{i}\right\} \mathrm{d} s$,

for all measurable selectors $(\zeta, \omega, \alpha)$ of $\left[-\frac{1}{2}, \frac{1}{2}\right] \times\left(1-\varepsilon_{i}\right)\left(\mathcal{C} \cap \mathbb{B}_{m}\right) \times A$;

(v) $\quad\left(m_{0_{i}}, m_{i}\right)(s) \in \partial_{t, x}^{>} h\left(y_{i}^{0}(s), y_{i}(s)\right) \quad \mu_{i}$-a.e. $s \in[0, \bar{S}]$,

(vi) $\operatorname{spt}\left(\mu_{i}\right) \subseteq\left\{s: h\left(y_{i}^{0}(s), y_{i}(s)\right)-c_{i}=0\right\}$,

where

$$
\left(q_{0_{i}}, q_{i}\right)(s):= \begin{cases}\left(p_{0_{i}}, p_{i}\right)(s)+\int_{[0, s[}\left(m_{0_{i}}, m_{i}\right)(\tau) \mu_{i}(d \tau) & s \in[0, \bar{S}[, \\ \left(p_{0_{i}}, p_{i}\right)(\bar{S})+\int_{[0, \bar{S}]}\left(m_{0_{i}}, m\right)(\tau) \mu_{i}(d \tau) & s=\bar{S} .\end{cases}
$$


Here $\tilde{L}$ is the same constant as in Eq. 24. By Banach-Alaoglu's Theorem, there exist a subsequence of $\left(\mu_{i}\right)_{i}, \mu \in N B V^{+}([0, \bar{S}] ; \mathbb{R}),\left(m_{0}, m\right):[0, \bar{S}] \rightarrow \mathbb{R} \times \mathbb{R}^{n}$ Borel measurable and $\mu$-integrable, such that $\mu_{i} \stackrel{*}{\rightarrow} \mu$ weakly* in $C^{*}([0, \bar{S}])$ and $m_{i} \mu_{i}(d s) \stackrel{*}{\rightarrow} m \mu(d s), m_{0_{i}} \mu_{i}(d s) \stackrel{*}{\rightarrow} m_{0} \mu(d s)$ (see [35, Proposition 9.2.1]). Furthermore, $\int_{[0, s[}\left(m_{0_{i}}, m_{i}\right)(\tau) \mu_{i}(d \tau) \rightarrow \int_{[0, s[}\left(m_{0}, m\right)(\tau) \mu(d \tau)$ for a.e. $s \in[0, \bar{S}]$; the real sequence $\left(\pi_{i}\right)$ is bounded; the functions $\left(p_{0_{i}}, p_{i}\right)$ are uniformly bounded and have uniformly integrable, bounded derivatives. Hence, there exist $\pi \leq 0$ and $\left(p_{0}, p\right) \in W^{1,1}\left([0, \bar{S}] ; \mathbb{R}^{1+n}\right)$ (see e.g. [35, Th 2.5.3 and Ch. 9]) such that, eventually for a further subsequence, $\pi_{i} \rightarrow \pi$, $\left(p_{0_{i}}, p_{i}\right) \rightarrow\left(p_{0}, p\right)$ in $L^{\infty}$, and $\left(\frac{d p_{0_{i}}}{d s}, \frac{d p_{i}}{d s}\right) \rightarrow\left(\frac{d p_{0}}{d s}, \frac{d p}{d s}\right)$ weakly in $L^{1}$, as $i \rightarrow+\infty$. By this analysis it also follows that the functions $\left(q_{0_{i}}, q_{i}\right)$ are uniformly integrably bounded and verify for a.e. $s \in[0, \bar{S}]$,

$$
\lim _{i \rightarrow+\infty}\left(q_{0_{i}}, q_{i}\right)(s)=\left(q_{0}, q\right)(s):=\left(p_{0}, p\right)(s)+\int_{[0, s[}\left(m_{0}, m\right)(\tau) \mu(d \tau) .
$$

Hence, by the dominated convergence theorem, one has

$$
\left(q_{0_{i}}, q_{i}\right) \rightarrow\left(q_{0}, q\right) \quad \text { in } L^{1}([0, \bar{S}]) .
$$

Passing to the limit as $i \rightarrow+\infty$ and using Eq. 17, by (i),(v), and (vi) we get

$$
\begin{gathered}
\left\|p_{0}\right\|_{L^{\infty}}+\|p\|_{L^{\infty}}+\|\mu\|_{T V}+|\pi|=1, \\
\left(m_{0}, m\right)(s) \in \partial_{t, x}^{>} h\left(\bar{y}^{0}(s), \bar{y}(s)\right) \quad \mu \text {-a.e. } s \in[0, \bar{S}], \\
\operatorname{spt}(\mu) \subseteq\left\{s: h\left(\bar{y}^{0}(s), \bar{y}(s)\right)=0\right\} .
\end{gathered}
$$

Furthermore, using that $\left(\bar{y}^{0}(0), \bar{y}(0), \bar{y}^{0}(\bar{S}), \bar{y}(\bar{S}), \bar{v}(\bar{S})\right) \in \mathcal{T}$, the properties of distance function, and the 'max-rule' for subdifferentials, by (iii) we have

$$
\begin{aligned}
& \left(p_{0}(0), p(0),-q_{0}(\bar{S}),-q(\bar{S}),-\pi\right) \in[0, \tilde{L}] \cdot \partial \Phi\left(\bar{y}^{0}(0), \bar{y}(0), \bar{y}^{0}(\bar{S}), \bar{y}(\bar{S}), \bar{v}(\bar{S})\right) \\
& \subseteq[0, \tilde{L}] \cdot\left[\left(N_{\mathcal{T}_{0}}\left(\bar{y}^{0}(0), \bar{y}(0), \bar{y}^{0}(\bar{S}), \bar{y}(\bar{S}), \bar{v}(\bar{S})\right) \cap \mathbb{B}_{1+n+1+n}\right) \times\{0\}\right. \\
& \left.+\left\{0_{1+n+1+n}\right\} \times \partial\left(\left(v_{i}(\bar{S})-K\right) \vee 0\right)\right] \\
& \subseteq N_{\mathcal{T}_{0}}\left(\bar{y}^{0}(0), \bar{y}(0), \bar{y}^{0}(\bar{S}), \bar{y}(\bar{S})\right) \times N_{-\infty, K]}(\bar{v}(\bar{S})),
\end{aligned}
$$

Incidentally, from this relation we immediately deduce that $\pi=0$ if $\bar{v}(\bar{S})<K$. Passing to the limit in (iv), with the help of a measurable selection theorem, using Eqs. 17 and 18 and the dominated convergence Theorem, we deduce that, for a.e. $s \in[0, \bar{S}]$,

$$
\begin{aligned}
& H\left(\bar{y}^{0}(s), \bar{y}(s), q_{0}(s), q(s), \pi, \bar{\omega}^{0}(s), \bar{\omega}(s), \bar{\alpha}(s)\right) \\
& \quad=\max _{\left(\zeta, \omega^{0}, \omega, a\right) \in\left[-\frac{1}{2}, \frac{1}{2}\right] \times \mathbf{C} \times A} H\left(\bar{y}^{0}(s), \bar{y}(s), q_{0}(s), q(s), \pi, \omega^{0}, \omega, a\right)(1+\zeta) .
\end{aligned}
$$

Since 0 is in the interior of $\left[-\frac{1}{2}, \frac{1}{2}\right]$, this implies that, for a.e. $s \in[0, \bar{S}]$,

$$
\begin{aligned}
H\left(\bar{y}^{0}(s), \bar{y}(s), q_{0}(s),\right. & \left.q(s), \pi, \bar{\omega}^{0}(s), \bar{\omega}(s), \bar{\alpha}(s)\right) \\
& =\max _{\left(\omega^{0}, \omega, a\right) \in \mathbf{C} \times A} H\left(\bar{y}^{0}(s), \bar{y}(s), q_{0}(s), q(s), \pi, \omega^{0}, \omega, a\right)=0 .
\end{aligned}
$$

To prove that $\left(p_{0}, p\right)$ verifies the adjoint equation in Theorem 2.1 , for each $i \in \mathbb{N}$ we set

$$
\mathcal{A}_{i}:=\left\{s \in[0, \bar{S}]:\left(\zeta_{i}(s), \alpha_{i}(s)\right)=(0, \bar{\alpha}(s))\right\} \subseteq[0, \bar{S}] .
$$


By Eq. 18 it follows that $\ell\left(\mathcal{A}_{i}\right) \rightarrow \bar{S}$ as $i \rightarrow+\infty$. Hence, by the $L$-boundedness of $f$ and $g_{1}, \ldots, g_{m}$, we deduce that the functions $\left(y_{i}^{0}, y_{i}\right)$ are uniformly integrable, so that by [35, Th. 2.5.3] and Eq. 17, $\left(\frac{d y_{i}^{0}}{d s}, \frac{d y_{i}}{d s}\right) \rightarrow\left(\frac{d y^{0}}{d s}, \frac{d y}{d s}\right)$ weakly in $L^{1}$, as $i \rightarrow+\infty$. Moreover, for a.e. $s \in \mathcal{A}_{i}$, we have

$$
\begin{aligned}
& \left(\frac{d y_{i}^{0}}{d s}, \frac{d y_{i}}{d s}\right)(s)=\left(\omega_{i}^{0}, f\left(y_{i}^{0}, y_{i}, \bar{\alpha}\right) \omega_{i}^{0}+\sum_{j=1}^{m} g_{j}\left(y_{i}^{0}, y_{i}\right) \omega_{i}^{j}\right)(s), \\
& \left(-\frac{d p_{0_{i}}}{d s},-\frac{d p_{i}}{d s}\right)(s) \in \operatorname{co} \partial_{t, x}\left\{q_{i}(s) \cdot\left(f\left(y_{i}^{0}, y_{i}, \bar{\alpha}\right) \omega_{i}^{0}+\sum_{j=1}^{m} g_{j}\left(y_{i}^{0}, y_{i}\right) \omega_{i}^{j}\right)(s)\right\},
\end{aligned}
$$

By Eqs. 17, 26, and [35, Theorem 2.5.3] we can conclude that, for a.e. $s \in[0, \bar{S}],{ }^{4}$

$$
\left(-\frac{d p_{0}}{d s},-\frac{d p}{d s}\right)(s) \in \operatorname{co} \partial_{t, x}\left\{q(s) \cdot\left(f\left(\bar{y}^{0}, \bar{y}, \bar{\alpha}\right) \bar{\omega}^{0}+\sum_{j=1}^{m} g_{j}\left(\bar{y}^{0}, \bar{y}\right) \bar{\omega}^{j}\right)(s)\right\} .
$$

In view of relations Eqs. 28, 29, 30, 31 and 32, to conclude the proof that the isolated feasible process $\left(\bar{S}, \bar{\omega}^{0}, \bar{\omega}, \bar{\alpha}, \bar{y}^{0}, \bar{y}, \bar{v}\right)$ is an abnormal extremal, it remains only to show that

$$
\left\|p_{0}\right\|_{L^{\infty}}+\|p\|_{L^{\infty}}+\|\mu\|_{T V} \neq 0
$$

Suppose by contradiction that Eq. 33 is not true. Then $q_{0} \equiv 0, q \equiv 0$ a.e., and by Eq. 27 we deduce that $\pi \neq 0$, which in turn implies $\bar{v}(\bar{S})=K>0$. Thanks to these information and integrating (31) in $[0, \bar{S}]$ we find that $0=\int_{0}^{\bar{S}} \pi|\bar{\omega}| \mathrm{d} s=\pi \bar{\nu}(\bar{S})=\pi K$, which is not possible.

Remark 3.1 (Multiple state constraints) In order to allow multiple state constraints $h_{i}$ for $i=1, \ldots, N$, it is sufficient replacing the payoff $\mathcal{J}$ in Eq. 13 by the function $\Phi\left(y^{0}(0), y(0), y^{0}(\bar{S}), y(\bar{S}), v(\bar{S})\right) \vee \max _{s \in[0, \bar{S}]} h_{1}\left(y^{0}(s), y(s)\right) \vee \cdots \vee$ $\max _{s \in[0, \bar{S}]} h_{N}\left(y^{0}(s), y(s)\right)$, and making obvious changes to the preceding proof.

\section{Nondegeneracy, Normality and No Infimum Gap}

As a consequence of Theorem 3.1, 'normality implies no infimum gap'. Precisely, as a corollary of the results in Section 3, we have:

Theorem 4.1 Assume hypotheses (H0)-(H2) are satisfied.

(i) Suppose that there exists an extended sense minimizer for $\left(\mathrm{P}_{e}\right)$ which is a normal extremal. Then there is no infimum gap.

(ii) Let $\left(\bar{S}, \bar{\omega}^{0}, \bar{\omega}, \bar{\alpha}, \bar{y}^{0}, \bar{y}, \bar{v}\right)$ be an extended sense local minimizer for the extended problem $\left(\mathrm{P}_{e}\right)$ which is a normal extremal. Then there is no local infimum gap at $\left(\bar{S}, \bar{\omega}^{0}, \bar{\omega}, \bar{\alpha}, \bar{y}^{0}, \bar{y}, \bar{v}\right)$.

As observed in the Introduction, the above 'normality test' is of more theoretical than practical interest (specially in the presence of state constraints). In this section we identify

\footnotetext{
${ }^{4}$ Notice that, since the convex hull of a compact subset of some space $\mathbb{R}^{k}$ is compact, our hypotheses on the vector fields $f, g_{1}, \ldots, g_{m}$ guarantee that the multifunction in the differential inclusion is closed.
} 
some verifiable conditions guaranteeing that every set of multipliers is normal. To begin with, let us introduce the notion of nondegenerate estremal.

Definition 4.1 (Nondegenerate Maximum Principle) Given an extended sense local minimizer for problem $\left(\mathrm{P}_{e}\right),\left(\bar{S}, \bar{\omega}^{0}, \bar{\omega}, \bar{\alpha}, \bar{y}^{0}, \bar{y}, \bar{v}\right)$, we say that the Maximum Principle is nondegenerate when there is a choice of the multipliers $\left(p_{0}, p, \pi, \lambda, \mu\right)$ and of the functions $m_{0}, m$ that meets the conditions (i)-(vi) of Theorem 2.1, and such that

$$
\begin{array}{lll}
\mu(] 0, \bar{S}])+\left\|q_{0}\right\|_{L^{\infty}}+\|q\|_{L^{\infty}}+\lambda \neq 0 & \text { if } & \bar{y}^{0}(\bar{S})=\bar{y}^{0}(0), \\
\mu(] 0, \bar{S}])+\|q\|_{L^{\infty}}+\lambda \neq 0 & \text { if } & \bar{y}^{0}(\bar{S})>\bar{y}^{0}(0),
\end{array}
$$

where $q_{0}, q$ are defined as in Theorem 2.1.

Definition 4.2 (Nondegenerate normal and abnormal extremals) We say that a feasible extended sense process $\left(\bar{S}, \bar{\omega}^{0}, \bar{\omega}, \bar{\alpha}, \bar{y}^{0}, \bar{y}, \bar{v}\right)$ is an extremal of the nondegenerate Maximum Principle, in short, a nondegenerate extremal, if there exists a set of multipliers $\left(p_{0}, p, \pi, \lambda, \mu\right)$ and functions $m_{0}, m$ which meet the conditions of Theorem 2.1 and also satisfy (34). We call $\left(\bar{S}, \bar{\omega}^{0}, \bar{\omega}, \bar{\alpha}, \bar{y}^{0}, \bar{y}, \bar{v}\right)$ a nondegenerate normal extremal if all possible choices of multipliers as above have $\lambda>0$, and a nondegenerate abnormal extremal when there exists at least one set of such multipliers with $\lambda=0$.

As it is easy to see, a nondegenerate abnormal extremal is always an abnormal extremal, and, on the contrary, any normal extremal is also nondegenerate normal. To obtain the converse implications, we introduce condition ( $\mathbf{C N a}$ ) below. In the following, we will often use the notation

$$
\Omega:=\{(t, x): h(t, x) \leq 0\}
$$

Condition for Nondegenerate Abnormality (CNa) . A feasible extended sense process $\left(\bar{S}, \bar{\omega}^{0}, \bar{\omega}, \bar{\alpha}, \bar{y}^{0}, \bar{y}, \bar{v}\right)$ is said to verify condition $(\mathbf{C N a})$ if

$$
\partial^{>} h\left(\bar{y}^{0}(0), \bar{y}(0)\right) \cap\left(-\operatorname{proj}_{\left(t_{1}, x_{1}\right)}\left(N_{\mathcal{T}_{0}}\left(\bar{y}^{0}(0), \bar{y}(0), \bar{y}^{0}(\bar{S}), \bar{y}(\bar{S})\right)\right)\right)=\emptyset .
$$

Remark 4.1 To clarify the geometrical meaning of condition (CNa), let us notice that, if $\left(\bar{y}^{0}(0), \bar{y}(0)\right) \in \operatorname{Int}(\Omega)$, condition (35) is trivially satisfied, since the hybrid subdifferential $\partial^{>} h\left(\bar{y}^{0}(0), \bar{y}(0)\right)=\emptyset$. Incidentally, observe that $\{(t, x): h(t, x)<0\} \subseteq \operatorname{Int}(\Omega)$ but the inclusion is in general strict. When instead $\left(\bar{y}^{0}(0), \bar{y}(0)\right) \in \partial \Omega$, Eq. 35 implies that $0 \notin \partial^{>} h\left(\bar{y}^{0}(0), \bar{y}(0)\right)$. If $h \in C^{2}$ in a neighborhood of $\left(\bar{y}^{0}(0), \bar{y}(0)\right) \in \partial \Omega$, Eq. 35 simply reads $\left(\nabla h\left(\bar{y}^{0}(0), \bar{y}(0)\right) \neq 0\right.$ and $)$

$$
\nabla h\left(\bar{y}^{0}(0), \bar{y}(0)\right) \notin-\operatorname{proj}_{\left(t_{1}, x_{1}\right)}\left(N_{\mathcal{T}_{0}}\left(\bar{y}^{0}(0), \bar{y}(0), \bar{y}^{0}(\bar{S}), \bar{y}(\bar{S})\right)\right) .
$$

Condition (36) is satisfied at $\left(\bar{y}^{0}(0), \bar{y}(0)\right)$ with $h\left(\bar{y}^{0}(0), \bar{y}(0)\right)=0$ and $\nabla h\left(\bar{y}^{0}(0), \bar{y}(0)\right) \neq 0$, when, for instance, $\mathcal{T}_{0}=\mathcal{T}_{1} \times \mathcal{T}_{2}$ with $\mathcal{T}_{1}, \mathcal{T}_{2}$ closed subsets of $\mathbb{R}^{1+n}$, $\mathcal{T}_{1} \subseteq \Omega$, and $N_{\mathcal{T}_{1}}\left(\bar{y}^{0}(0), \bar{y}(0)\right)$ is pointed. ${ }^{5}$ In this case, indeed, Eq. 36 can be derived by the following relations

$$
\begin{aligned}
\partial^{>} h\left(\bar{y}^{0}(0), \bar{y}(0)\right) & =\left\{\nabla h\left(\bar{y}^{0}(0), \bar{y}(0)\right)\right\} \subseteq N_{\Omega}^{P}\left(\bar{y}^{0}(0), \bar{y}(0)\right) \\
& \subseteq N_{\mathcal{T}_{1}}^{P}\left(\bar{y}^{0}(0), \bar{y}(0)\right) \subseteq N_{\mathcal{T}_{1}}\left(\bar{y}^{0}(0), \bar{y}(0)\right) .
\end{aligned}
$$

\footnotetext{
${ }^{5}$ A cone $\mathcal{K} \subseteq \mathbb{R}^{k}$ is pointed if it contains no line, i.e. if $\xi,-\xi \in \mathcal{K}$ implies that $\xi=0$.
} 
Remark 4.2 Consider the quite customary situation where initial and final time are fixed and the state constraint is time independent, namely $\mathcal{T}_{0}=\left\{\bar{t}_{1}\right\} \times \mathcal{T}_{0}^{1} \times \mathcal{T}_{0}^{2}$ with $\mathcal{T}_{0}^{1} \subseteq \mathbb{R}^{n}$, $\mathcal{T}_{0}^{2} \subseteq \mathbb{R}^{1+n}$ closed subsets, and $h(t, x)=\bar{h}(x)$. In this case, $\partial^{>} h(t, x)=\{0\} \times \partial^{>} \bar{h}(x)$ and $N_{\left\{\bar{t}_{1}\right\} \times \mathcal{T}_{0}^{1}}(t, x)=\mathbb{R} \times N_{\mathcal{T}_{0}^{1}}(x)$ for all $(t, x) \in \mathbb{R}^{1+n}$. Hence, condition (35) reduces to

$$
\partial^{>} \bar{h}(\bar{y}(0)) \cap\left(-N_{\mathcal{T}_{0}^{1}}(\bar{y}(0))\right)=\emptyset .
$$

Proposition 4.1 Assume (H0)-(H2) and suppose that $\left(\bar{S}, \bar{\omega}^{0}, \bar{\omega}, \bar{\alpha}, \bar{y} 0, \bar{y}, \bar{v}\right)$ is a feasible extended sense process which is an abnormal extremal, namely, there exist a set of multipliers $\left(p_{0}, p, \pi, \lambda, \mu\right)$, and some functions $\left(m_{0}, m\right)$ as in Theorem 2.1 , with $\lambda=0$. If condition (CNa) is satisfied, then $\left(p_{0}, p, \pi, 0, \mu\right),\left(m_{0}, m\right)$ verify the strengthened non-triviality condition (34).

Proof Assume that $(\mathbf{C N a})$ is verified and suppose by contradiction that $\left\|q_{0}\right\|_{L^{\infty}}+\|q\|_{L^{\infty}}+$ $\mu(] 0, \bar{S}])=0$. Then, in view of Theorem 2.1 , we have

$$
\left(p_{0}, p\right) \equiv-\mu(\{0\})\left(\xi_{0}, \xi\right), \quad \mu(\{0\}) \neq 0, \quad\left(\xi_{0}, \xi\right) \in \partial^{>} h\left(\bar{y}^{0}(0), \bar{y}(0)\right)
$$

In particular, $\mu(\{0\}) \neq 0$ implies that $\left(\bar{y}^{0}(0), \bar{y}(0)\right) \in \partial \Omega$. By the transversality condition (iii) of Theorem 2.1, it follows that

$$
\left(\xi_{0}, \xi\right) \in \partial^{>} h\left(\bar{y}^{0}(0), \bar{y}(0)\right) \cap\left(-\operatorname{proj}_{\left(t_{1}, x_{1}\right)}\left(N_{\mathcal{T}_{0}}\left(\bar{y}^{0}(0), \bar{y}(0), \bar{y}^{0}(\bar{S}), \bar{y}(\bar{S})\right)\right)\right),
$$

in contradiction with Eq. 35 .

To conclude it remains to show that $\left.\left.\|q\|_{L^{\infty}}+\mu(] 0, \bar{S}\right]\right) \neq 0$ whenever $\bar{y}^{0}(\bar{S})>\bar{y}^{0}(0)$. If we suppose by contradiction $\mu(] 0, \bar{S}])+\|q\|_{L^{\infty}}=0$, then Theorem 2.1, (ii), (iv) and the first part of the proof yield that $0 \neq q_{0}$ is a constant and

$$
\left.q_{0} \bar{w}^{0}(s)+\pi|\bar{w}(s)|=\max _{w^{0} \in[0,1]}\left\{\pi+\left(q_{0}-\pi\right) w^{0}\right\}=0 \quad \text { a.e. } s \in\right] 0, \bar{S}[.
$$

Then $\pi<0$ leads easily to a contradiction. If $\pi=0$, since $q_{0} \neq 0$, Eq. 38 yields $\bar{w}^{0}=0$ a.e., in contradiction with $\int_{0}^{\bar{S}} \bar{w}^{0}(s) d s=\bar{y}^{0}(\bar{S})-\bar{y}^{0}(0)>0$.

As a straightforward consequence of Proposition 4.1, we have:

Proposition 4.2 Assume (H0)-(H2). Let $\left(\bar{S}, \bar{\omega}^{0}, \bar{\omega}, \bar{\alpha}, \bar{y}^{0}, \bar{y}, \bar{v}\right)$ be a feasible extended sense process verifying condition $(\mathbf{C N a})$. Then $\left(\bar{S}, \bar{\omega}^{0}, \bar{\omega}, \bar{\alpha}, \bar{y}^{0}, \bar{y}, \bar{v}\right)$ is a nondegenerate normal extremal if and only if it is a normal extremal.

Remark 4.3 (Multiple state constraints) The result of Proposition 4.1 can be easily extended to the case of multiple state constraints $h_{i}$ for $i=1, \ldots, N$, by considering the nondegener- 
acy condition (34) with $\left(q_{0}, q\right)$ and $\mu$ as in Remark 2.4 and by replacing the nondegenerate abnormality condition (35) with

$$
\begin{aligned}
& \left(\sum _ { i = 1 } ^ { N } \left[0,+\infty\left[\cdot \partial^{>} h_{i}\left(\bar{y}^{0}(0), \bar{y}(0)\right)\right)\right.\right. \\
& \cap\left(-\operatorname{proj}_{\left(t_{1}, x_{1}\right)}\left(N_{\mathcal{T}_{0}}\left(\bar{y}^{0}(0), \bar{y}(0), \bar{y}^{0}(\bar{S}), \bar{y}(\bar{S})\right)\right)\right)=\emptyset .
\end{aligned}
$$

We now provide some sufficient conditions in the form of constraint and endpoint qualifications to guarantee normality. In the following, we will use the notation

$$
F\left(t, x, \omega^{0}, \omega, a\right):=f(t, x, a) \omega^{0}+\sum_{i=1}^{m} g_{i}(t, x) \omega^{i} \quad \forall\left(t, x, \omega^{0}, \omega, a\right) \in \mathbb{R}^{1+n} \times \mathbf{C} \times A,
$$

and, given a feasible extended sense process $\left(\bar{S}, \bar{\omega}^{0}, \bar{\omega}, \bar{\alpha}, \bar{y}^{0}, \bar{y}, \bar{v}\right)$ for problem $\left(\mathrm{P}_{e}\right)$, we set

$$
\bar{F}(s):=F\left(\left(\bar{y}^{0}, \bar{y}, \bar{\omega}^{0}, \bar{\omega}, \bar{\alpha}\right)(s)\right) \quad \forall s \in[0, \bar{S}], \quad \bar{z}:=\left(\bar{S}, \bar{\omega}^{0}, \bar{\omega}, \bar{\alpha}, \bar{y}^{0}, \bar{y}, \bar{v}\right) .
$$

Constraint Qualifications for Normality $(\mathrm{CQn})_{b},(\mathrm{CQn})_{f}$ Let $\bar{z}$ be a feasible extended sense process for the extended optimization problem $\left(\mathrm{P}_{e}\right)$.

$(\mathbf{C Q n})_{b}$ We say that $\bar{z}$ meets condition (CQn) $)_{b}$ if for every $\left.\left.s \in\right] 0, \bar{S}\right]$ where $\left(\bar{y}^{0}(s), \bar{y}(s)\right) \in \partial \Omega$ there exist $\varepsilon, \delta>0$ such that ${ }^{7}$

$$
\begin{gathered}
\inf _{a \in A} \max _{\left(\xi_{0}, \xi\right) \in \partial^{*} h\left(\bar{y}^{0}(s), \bar{y}(s)\right)}\left[\xi \cdot\left(f\left(\bar{y}^{0}(s), \bar{y}(s), a\right)-f\left(\bar{y}^{0}(s), \bar{y}(s), \bar{\alpha}(\sigma)\right)\right)\right]<-\delta, \\
\inf _{\omega \in \mathcal{C} \cap \partial \mathbb{B}} \max _{\left(\xi_{0}, \xi\right) \in \partial^{*} h\left(\bar{y}^{0}(s), \bar{y}(s)\right)}\left[\xi \cdot\left(\sum_{j=1}^{m} g_{j}\left(\bar{y}^{0}(s), \bar{y}(s)\right)\left(\omega^{j}-\frac{\bar{\omega}^{j}(\sigma)}{\left|\bar{\omega}^{j}(\sigma)\right|}\right)\right]<-\delta\right.
\end{gathered}
$$

for a.e. $\sigma \in E(s, \varepsilon)$, defined as follows

$$
E(s, \varepsilon):=\left\{r \in[s-\varepsilon, s] \cap[0, \bar{S}]: \max _{\left(\xi_{0}, \xi\right) \in \partial^{*} h\left(\bar{y}^{0}(r), \bar{y}(r)\right)}\left(\xi_{0} \bar{\omega}^{0}+\xi \cdot \bar{F}\right)(r) \geq 0\right\} ;
$$

(CQn) $f \quad$ We say that $\bar{z}$ meets condition (CQn) $f$ if for every $s \in[0, \bar{S}[$ where $\left(\bar{y}^{0}(s), \bar{y}(s)\right) \in \partial \Omega$ there exist $\varepsilon, \delta>0$ such that

$$
\begin{gathered}
\sup _{a \in A} \min _{(\xi 0, \xi) \in \partial^{*} h\left(\bar{y}^{0}(s), \bar{y}(s)\right)}\left[\xi \cdot\left(f\left(\bar{y}^{0}(s), \bar{y}(s), a\right)-f\left(\bar{y}^{0}(s), \bar{y}(s), \bar{\alpha}(\sigma)\right)\right)\right]>\delta, \\
\sup _{\omega \in \mathcal{C} \cap \partial \mathbb{B}\left(\xi_{0}, \xi\right) \in \partial^{*} h\left(\bar{y}^{0}(s), \bar{y}(s)\right)}\left[\xi \cdot\left(\sum_{j=1}^{m} g_{j}\left(\bar{y}^{0}(s), \bar{y}(s)\right)\left(\omega^{j}-\frac{\bar{\omega}^{j}(\sigma)}{\left|\bar{\omega}^{j}(\sigma)\right|}\right)\right]>\delta\right.
\end{gathered}
$$

for a.e. $\sigma \in \Gamma(s, \varepsilon)$, defined as follows

$$
\Gamma(s, \varepsilon):=\left\{r \in[s, s+\varepsilon] \cap[0, \bar{S}]: \min _{\left(\xi_{0}, \xi\right) \in \partial^{*} h\left(\bar{y}^{0}(r), \bar{y}(r)\right)}\left(\xi_{0} \bar{\omega}^{0}+\xi \cdot \bar{F}\right)(r) \leq 0\right\} .
$$

Remark 4.4 The 'forward' constraint qualification (CQn) $f$ is at our knowledge new, while a version of the 'backward' constraint qualification (CQn) $)_{b}$ was first introduced in [25], as an adaptation to impulsive optimal control of a condition due to [13]. In particular,

\footnotetext{
${ }^{6}$ To simplify the notation, when $X \subseteq \mathbb{R}^{k}$, we use the convention $X+\emptyset=X$. Thus, in particular, $\emptyset+\emptyset=\emptyset$.

${ }^{7}$ We use the convention that $\frac{\omega}{|\omega|}=0$ when $\omega=0$.
} 
$(\mathbf{C Q n})_{b},(\mathbf{C Q n})_{f}$ prescribe that drift and fast dynamics satisfy separately outward or inward pointing conditions, respectively. This is a stronger requirement than the existence of an inward/outward pointing velocity. In fact, as discussed in [25, Remark 4.4], the statement of Theorem 4.3 below holds true even if $(\mathbf{C Q n})_{b},(\mathbf{C Q n})_{f}$ are replaced, respectively, with the (weaker) conditions:

$(\mathbf{C Q n})_{b}^{\prime}$ for every $\left.\left.s \in\right] 0, \bar{S}\right]$ such that $\left(\bar{y}^{0}(s), \bar{y}(s)\right) \in \partial \Omega$ there exist $\varepsilon, \delta>0$ and a measurable control $(\tilde{\omega}, \hat{\alpha})$ taking values in $(\mathcal{C} \cap \partial \mathbb{B}) \times A$, satisfying for all $\sigma \in] s-\varepsilon, s[\cap[0, \bar{S}]:$

$$
\max _{\left(\xi_{0}, \xi\right) \in \partial^{*} h\left(\bar{y}^{0}(\sigma), \bar{y}(\sigma)\right)} \xi \cdot\left(F\left(\bar{y}^{0}, \bar{y}, \bar{\omega}^{0}, \hat{\omega}, \hat{\alpha}\right)\left(s^{\prime}\right)-\bar{F}\left(s^{\prime}\right)\right)<-\delta,
$$

where $\hat{\omega}:=\left(1-\bar{\omega}^{0}\right) \tilde{\omega}$, for a.e. $s^{\prime} \in E(s, \varepsilon)$, defined as in $(\mathbf{C Q n})_{b}$;

$(\mathbf{C Q n})_{f}^{\prime} \quad$ for every $s \in\left[0, \bar{S}\left[\right.\right.$ such that $\left(\bar{y}^{0}(s), \bar{y}(s)\right) \in \partial \Omega$ there exist $\varepsilon, \delta>0$ and a measurable control $(\tilde{\omega}, \hat{\alpha})$ taking values in $(\mathcal{C} \cap \partial \mathbb{B}) \times A$, satisfying for all $\sigma \in] s, s+\varepsilon[\cap[0, \bar{S}]:$

$$
\min _{\left(\xi^{0}, \xi\right) \in \partial^{*} h\left(\bar{y}^{0}(\sigma), \bar{y}(\sigma)\right)} \xi \cdot\left(F\left(\bar{y}^{0}, \bar{y}, \bar{\omega}^{0}, \hat{\omega}, \hat{\alpha}\right)\left(s^{\prime}\right)-\bar{F}\left(s^{\prime}\right)\right)>\delta,
$$

where $\hat{\omega}:=\left(1-\bar{\omega}^{0}\right) \tilde{w}$, for a.e. $s^{\prime} \in \Gamma(s, \varepsilon)$, defined as in (CQn) $f$.

Notice that conditions (CQn) ${ }_{b}^{\prime}$, (CQn) ${ }_{f}^{\prime}$ also cover situations in which the drift does not depend on the ordinary control $a$, i.e. $f=f(t, x)$, unlike $(\mathbf{C Q n})_{b},(\mathbf{C Q n})_{f}$, where in this case the hypotheses involving $f$ are clearly never fulfilled.

Remark 4.5 The constraint qualifications $(\mathbf{C Q n})_{b},(\mathbf{C Q n})_{f}$ are respectively inward/outward pointing conditions at the boundary which involve the minimizer but have to be satisfied just on a subset of instants at which the optimal trajectory has an outward/inward pointing velocity. As discussed in detail in [25, Lemma 4.1], when the constraint function $h$ is smooth, $(\mathbf{C Q n})_{b},(\mathbf{C Q n})_{f}$ could be replaced by the following simpler conditions:

$(\text { IPFCn })_{b}$ for every $\left.\left.s \in\right] 0, \bar{S}\right]$ such that $\left(\bar{y}^{0}(s), \bar{y}(s)\right) \in \partial \Omega$, one has $h \in C^{1}$ on a neighborhood of $\left(\bar{y}^{0}(s), \bar{y}(s)\right)$ and there exists $\delta>0$ satisfying

$$
\begin{aligned}
& \inf _{a \in A} \nabla_{x} h\left(\bar{y}^{0}(s), \bar{y}(s)\right) \cdot f\left(\bar{y}^{0}(s), \bar{y}(s), a\right)<-\delta, \\
& \inf _{\omega \in \mathcal{C} \cap \partial \mathbb{B}} \nabla_{x} h\left(\bar{y}^{0}(s), \bar{y}(s)\right) \cdot \sum_{i=1}^{m} g_{i}\left(\bar{y}^{0}(s), \bar{y}(s)\right) \omega^{i}<-\delta ;
\end{aligned}
$$

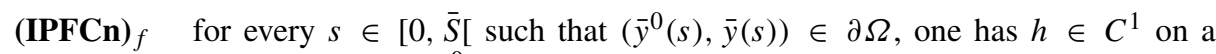
neighborhood of $\left(\bar{y}^{0}(s), \bar{y}(s)\right)$ and there exists $\delta>0$ satisfying

$$
\begin{aligned}
& \sup _{a \in A} \nabla_{x} h\left(\bar{y}^{0}(s), \bar{y}(s)\right) \cdot f\left(\bar{y}^{0}(s), \bar{y}(s), a\right)>\delta, \\
& \sup _{\omega \in \mathcal{C} \cap \partial \mathbb{B}} \nabla_{x} h\left(\bar{y}^{0}(s), \bar{y}(s)\right) \cdot \sum_{i=1}^{m} g_{i}\left(\bar{y}^{0}(s), \bar{y}(s)\right) \omega^{i}>\delta,
\end{aligned}
$$

respectively. Here $\nabla_{x}$ denotes the classical partial gradient operator w.r.t. the variable $x$.

Remark 4.6 Note that for a feasible process $\bar{z}=\left(\bar{S}, \bar{\omega}^{0}, \bar{\omega}, \bar{\alpha}, \bar{y} 0, \bar{y}, \bar{v}\right)$ with $h\left(\bar{y}^{0}(\bar{s}), \bar{y}(\bar{s})\right)=0$ for some $\left.\left.\bar{s} \in\right] 0, \bar{S}\right]$, we can assume that there exists some $\bar{\varepsilon}_{1}>0$ sufficiently small such that $\ell(E(\bar{s}, \varepsilon))>0$ for all $\left.\varepsilon \in] 0, \bar{\varepsilon}_{1}\right]$. Indeed, if $\exists \varepsilon>0$ such that $\ell(E(\bar{s}, \varepsilon))=0$, then $\max _{\left(\xi_{0}, \xi\right) \in \partial^{*} h\left(\bar{y}^{0}(r), \bar{y}(r)\right)}\left[\xi_{0} \bar{\omega}^{0}(r)+\xi \cdot \bar{F}(r)\right]<0$ for a.e. $r \in[\bar{s}-\varepsilon, \bar{s}]$. 
But then, the function $\mathcal{H}:=h \circ\left(\bar{y}^{0}, \bar{y}\right)$ is differentiable a.e. in $[\bar{s}-\varepsilon, \bar{s}]$ and verifies

$$
\begin{aligned}
\frac{d \mathcal{H}}{d s}(s) & \leq \max _{\left(\xi_{0}, \xi\right) \in \partial^{C} h\left(\bar{y}^{0}(s), \bar{y}(s)\right)}\left[\xi_{0} \frac{d \bar{y}^{0}}{d s}(s)+\xi \cdot \frac{d \bar{y}}{d s}(s)\right] \\
& =\max _{\left(\xi_{0}, \xi\right) \in \partial^{*} h\left(\bar{y}^{0}(s), \bar{y}(s)\right)}\left[\xi_{0} \bar{\omega}^{0}(s)+\xi \cdot \bar{F}(s)\right]<0,
\end{aligned}
$$

since the scalar product is bilinear. Thus, for all $s \in[\bar{s}-\varepsilon, \bar{s}[$ one has

$$
-h\left(\bar{y}^{0}(s), \bar{y}(s)\right)=h\left(\bar{y}^{0}(\bar{s}), \bar{y}(\bar{s})\right)-h\left(\bar{y}^{0}(s), \bar{y}(s)\right)=\int_{s}^{\bar{s}} \frac{d \mathcal{H}}{d s}(\sigma) d \sigma<0,
$$

so that $h\left(\bar{y}^{0}(s), \bar{y}(s)\right)>0$, in contradiction with the feasibility of $\bar{z}$. In an analogous way, one can derive that, if $h\left(\bar{y}^{0}(\bar{s}), \bar{y}(\bar{s})\right)=0$ at $\bar{s} \in[0, \bar{S}[$, then $\ell(\Gamma(\bar{s}, \varepsilon))>0$ for all $\varepsilon>0$ small enough.

Furthermore, by the bilinearity of the scalar product, in $(\mathbf{C Q n})_{b},(\mathbf{C Q n})_{f}$, and (CQn) ${ }_{b}^{\prime}, \quad(\mathbf{C Q n})_{f}^{\prime}$, one can replace $\partial^{*} h\left(\bar{y}^{0}(s), \bar{y}(s)\right)$ with $\partial^{C} h\left(\bar{y}^{0}(s), \bar{y}(s)\right)$. Hence, in particular, all these conditions are verified for any $\left(\xi_{0}, \xi\right) \in$ $\partial^{>} h\left(\bar{y}^{0}(s), \bar{y}(s)\right)$, since $\partial^{>} h\left(\bar{y}^{0}(s), \bar{y}(s)\right) \subseteq \partial^{C} h\left(\bar{y}^{0}(s), \bar{y}(s)\right)$.

We can now establish the following normal Maximum Principle, which extends [25, Th. 4.2].

Theorem 4.2 Assume (H0)-(H2). Let $\left(\bar{S}, \bar{\omega}^{0}, \bar{\omega}, \bar{\alpha}, \bar{y}^{0}, \bar{y}, \bar{v}\right)$ be an extended sense local minimizer for $\left(\mathrm{P}_{e}\right)$. Assume that $\left(p_{0}, p, \lambda, \pi, \mu\right),\left(m_{0}, m\right)$ meet the conditions of Theorem 2.1 and verify the strenghtened non-triviality condition (34). Then,

(i) if hypothesis $(\mathbf{C Q n})_{b}$ is satisfied, one has

$$
\begin{array}{ll}
|q(\bar{S})|+\lambda \neq 0 & \text { if } \bar{y}^{0}(\bar{S})>\bar{y}^{0}(0), \\
\left|q_{0}(\bar{S})\right|+|q(\bar{S})|+\lambda \neq 0 & \text { if } \bar{y}^{0}(\bar{S})=\bar{y}^{0}(0) .
\end{array}
$$

In particular, if $\operatorname{proj}_{\left(t_{2}, x_{2}\right)} N_{\mathcal{T}_{0}}\left(\bar{y}^{0}(0), \bar{y}(0), \bar{y}^{0}(\bar{S}), \bar{y}(\bar{S})\right)=\left\{\left(\xi_{t_{2}}, 0\right)\right\}$ and $\xi_{t_{2}}=0$ whenever $\bar{y}^{0}(\bar{S})=\bar{y}^{0}(0)$, then $\lambda \neq 0$;

(ii) if hypothesis $(\mathbf{C Q n})_{f}$ is satisfied, one has

$$
\begin{array}{ll}
|q(0)|+\lambda \neq 0 & \text { if } \bar{y}^{0}(\bar{S})>\bar{y}^{0}(0), \\
\left|q_{0}(0)\right|+|q(0)|+\lambda \neq 0 & \text { if } \bar{y}^{0}(\bar{S})=\bar{y}^{0}(0) .
\end{array}
$$

In particular, if $\operatorname{proj}_{\left(t_{1}, x_{1}\right)} N_{\mathcal{T}_{0}}\left(\bar{y}^{0}(0), \bar{y}(0), \bar{y}^{0}(\bar{S}), \bar{y}(\bar{S})\right)=\left\{\left(\xi_{t_{1}}, 0\right)\right\}$ and $\xi_{t_{1}}=0$ whenever $\bar{y}^{0}(\bar{S})=\bar{y}^{0}(0)$, then $\lambda \neq 0$.

The proof of this result is given in the Appendix.

Remark 4.7 (Multiple state constraints) The normal Maximum Principle in Theorem 4.2 can be extended to the case with multiple state constraints $h_{i}$ for $i=1, \ldots, N$, by modifying condition (CQn) ${ }_{b}^{\prime}$ as follows: for every $\left.\left.s \in\right] 0, \bar{S}\right]$ such that $h_{i_{j}}\left(\bar{y}^{0}(s), \bar{y}(s)\right)=0$ for some indexes $1 \leq i_{1}<\cdots<i_{k} \leq N, j=1, \ldots, k$, there exist $\varepsilon, \delta>0$ and a measurable control $(\tilde{\omega}, \hat{\alpha})$ taking values in $(\mathcal{C} \cap \partial \mathbb{B}) \times A$, satisfying for all $\sigma \in] s-\varepsilon, s[\cap[0, \bar{S}]$ and for all $j=1, \ldots, k$ :

$$
\max _{\left(\xi_{0}, \xi\right) \in \partial^{*} h_{i_{j}}\left(\bar{y}^{0}(\sigma), \bar{y}(\sigma)\right)} \xi \cdot\left(F\left(\bar{y}^{0}, \bar{y}, \bar{\omega}^{0}, \hat{\omega}, \hat{\alpha}\right)\left(s^{\prime}\right)-\bar{F}\left(s^{\prime}\right)\right)<-\delta,
$$


where $\hat{\omega}:=\left(1-\bar{\omega}^{0}\right) \tilde{\omega}$, for a.e. $s^{\prime} \in \mathcal{E}(s, \varepsilon)$, defined as follows

$$
\begin{aligned}
& \mathcal{E}(s, \varepsilon):=\{r \in[s-\varepsilon, s] \cap[0, \bar{S}]: \\
&\left.\max _{\left(\xi_{0}, \xi\right) \in \partial^{*} h_{i_{j}}\left(\bar{y}^{0}(r), \bar{y}(r)\right)}\left[\xi_{0} \bar{\omega}^{0}(r)+\xi \cdot \bar{F}(r)\right] \geq 0 \quad \forall j=1, \ldots, k\right\} .
\end{aligned}
$$

Notice that, arguing as in Remark 4.6, one can deduce that $\ell(\mathcal{E}(s, \varepsilon)$ ) for all $\varepsilon>0$ sufficiently small. Similar modifications are to be made for condition $(\mathbf{C Q n})_{f}^{\prime}$.

Theorem 4.2 implies nondegenerate normality when essentially the endpoint constraint either at the final or at the initial position is inactive. We provide below some sufficient conditions to guarantee normality even in some situations where initial and final positions lay on the boundary of the endpoint constraint.

Endpoint Qualifications for Normality $(T Q n)_{b}$, $(T Q n)_{f}$. Let us consider $\bar{z}:=$ $\left(\bar{S}, \bar{\omega}^{0}, \bar{\omega}, \bar{\alpha}, \bar{y}^{0}, \bar{y}, \bar{v}\right)$, a feasible extended sense process for the extended optimization problem $\left(\mathrm{P}_{e}\right)$.

$(\text { TQn })_{b}$ We say that $\bar{z}$ meets condition (TQn) $)_{b}$ if there exists $\varepsilon>0$ such that $\left(\bar{y}^{0}(s), \bar{y}(s)\right) \in \operatorname{Int}(\Omega)$ for each $s \in[\bar{S}-\varepsilon, \bar{S}[$ and one among the following conditions (a), (b) holds true:

$$
\begin{aligned}
\text { (a) } & \left(-\operatorname{proj}_{\left(t_{2}, x_{2}\right)} N_{\mathcal{T}_{0}}\left(\bar{y}^{0}(0), \bar{y}(0), \bar{y}^{0}(\bar{S}), \bar{y}(\bar{S})\right) \backslash\left\{0_{1+n}\right\}\right) \\
& \cap \partial^{>} h\left(\bar{y}^{0}(S), \bar{y}(\bar{S})\right)=\emptyset
\end{aligned}
$$

and for any $\left(\zeta_{t_{2}}, \zeta_{x_{2}}\right) \in \operatorname{proj}_{\left(t_{2}, x_{2}\right)} N_{\mathcal{T}_{0}}\left(\bar{y}^{0}(0), \bar{y}(0), \bar{y}^{0}(\bar{S}), \bar{y}(\bar{S})\right)$ $+\left[0,+\infty\left[\cdot \partial^{>} h\left(\bar{y}^{0}(\bar{S}), \bar{y}(\bar{S})\right)\right.\right.$, one has

$$
\begin{gathered}
\min _{a \in A}\left[\zeta_{x_{2}} \cdot f\left(\bar{y}^{0}(\bar{S}), \bar{y}(\bar{S}), a\right)+\zeta_{t_{2}}\right]<0 \quad \text { if }\left(\zeta_{t_{2}}, \zeta_{x_{2}}\right) \neq(0,0) ; \\
\text { (b) }\left(-\operatorname{proj}_{x_{2}} N_{\mathcal{T}_{0}}\left(\bar{y}^{0}(0), \bar{y}(0), \bar{y}^{0}(\bar{S}), \bar{y}(\bar{S})\right) \backslash\left\{0_{n}\right\}\right) \\
\cap \operatorname{proj}_{x} \partial^{>} h\left(\bar{y}^{0}(\bar{S}), \bar{y}(\bar{S})\right)=\emptyset
\end{gathered}
$$

and for any $\left(\zeta_{t_{2}}, \zeta_{x_{2}}\right) \in \operatorname{proj}_{\left(t_{2}, x_{2}\right)} N_{\mathcal{T}_{0}}\left(\bar{y}^{0}(0), \bar{y}(0), \bar{y}^{0}(\bar{S}), \bar{y}(\bar{S})\right)$ $+\left[0,+\infty\left[\cdot \partial^{>} h\left(\bar{y}^{0}(\bar{S}), \bar{y}(\bar{S})\right)\right.\right.$ with $\zeta_{x_{2}} \neq 0$, one has

$$
\bar{y}^{0}(\bar{S})>\bar{y}^{0}(0), \quad \bar{v}(\bar{S})<K, \min _{\omega \in \mathcal{C} \cap \partial \mathbb{B}}\left[\zeta_{x_{2}} \cdot\left(\sum_{j=1}^{m} g_{j}\left(\bar{y}^{0}(\bar{S}), \bar{y}(\bar{S})\right) \omega^{j}\right)\right]<0 .
$$

(TQn) $f$ We say that $\bar{z}$ meets condition (TQn) $f$ if there exists $\varepsilon>0$ such that $\left(\bar{y}^{0}(s), \bar{y}(s)\right) \in \operatorname{Int}(\Omega)$ for each $\left.\left.s \in\right] 0, \varepsilon\right]$ and one among the following conditions (a), (b) holds true:

$$
\text { (a) } \begin{aligned}
\left(-\operatorname{proj}_{\left(t_{1}, x_{1}\right)} N_{\mathcal{T}_{0}}\left(\bar{y}^{0}(0), \bar{y}(0), \bar{y}^{0}(\bar{S}), \bar{y}(\bar{S})\right) \backslash\left\{0_{1+n}\right\}\right) \\
\cap \partial^{>} h\left(\bar{y}^{0}(0), \bar{y}(0)\right)=\emptyset
\end{aligned}
$$

and for any $\left(\zeta_{t_{1}}, \zeta_{x_{1}}\right) \in \operatorname{proj}_{\left(t_{1}, x_{1}\right)} N_{\mathcal{T}_{0}}\left(\bar{y}^{0}(0), \bar{y}(0), \bar{y}^{0}(\bar{S}), \bar{y}(\bar{S})\right)$ $+\left[0,+\infty\left[\cdot \partial^{>} h\left(\bar{y}^{0}(0), \bar{y}(0)\right)\right.\right.$ one has

$$
\max _{a \in A}\left[\zeta_{x_{1}} \cdot f\left(\bar{y}^{0}(0), \bar{y}(0), a\right)+\xi_{t_{1}}\right]>0 \quad \text { if }\left(\zeta_{t_{1}}, \zeta_{x_{1}}\right) \neq(0,0)
$$




$$
\text { (b) } \begin{aligned}
&\left(-\operatorname{proj}_{x_{1}} N_{\mathcal{T}_{0}}\left(\bar{y}^{0}(0), \bar{y}(0), \bar{y}^{0}(\bar{S}), \bar{y}(\bar{S})\right) \backslash\left\{0_{n}\right\}\right) \\
& \cap \operatorname{proj}_{x} \partial^{>} h\left(\bar{y}^{0}(0), \bar{y}(0)\right)=\emptyset
\end{aligned}
$$

and for any $\left(\zeta_{t_{1}}, \zeta_{x_{1}}\right) \in \operatorname{proj}_{\left(t_{1}, x_{1}\right)} N_{\mathcal{T}_{0}}\left(\bar{y}^{0}(0), \bar{y}(0), \bar{y}^{0}(\bar{S}), \bar{y}(\bar{S})\right)$ $+\left[0,+\infty\left[\cdot \partial^{>} h\left(\bar{y}^{0}(0), \bar{y}(0)\right)\right.\right.$ with $\zeta_{x_{1}} \neq 0$, one has

$$
\bar{y}^{0}(\bar{S})>\bar{y}^{0}(0), \quad \bar{v}(\bar{S})<K, \quad \max _{\omega \in \mathcal{C} \cap \partial \mathbb{B}} \zeta_{x_{1}} \cdot \sum_{j=1}^{m} g_{j}\left(\bar{y}^{0}(0), \bar{y}(0)\right) \omega^{j}>0 .
$$

Condition (TQn) ${ }_{b}$ generalizes endpoint constraint qualifications considered in [25] for the case with fixed initial point, which were in turn inspired by no gap conditions in $[1,26]$. Notice that both conditions Eqs. 45 and 47 [resp., Eqs. 49 and 51] are trivially verified whenever $\left(\bar{y}^{0}(\bar{S}), \bar{y}(\bar{S})\right) \in \operatorname{Int}(\Omega)\left[\operatorname{resp} .,\left(\bar{y}^{0}(0), \bar{y}(0)\right) \in \operatorname{Int}(\Omega)\right]$, since $\partial^{>} h\left(\bar{y}^{0}(\bar{S}), \bar{y}(\bar{S})\right)=\emptyset$ [resp., $\left.\partial^{>} h\left(\bar{y}^{0}(0), \bar{y}(0)\right)=\emptyset\right]$.

Proposition 4.3 Assume (H0)-(H2). Let $\left(\bar{S}, \bar{\omega}^{0}, \bar{\omega}, \bar{\alpha}, \bar{y}^{0}, \bar{y}, \bar{v}\right)$ be an extended sense local minimizer for $\left(\mathrm{P}_{e}\right)$. Assume that $\left(p_{0}, p, \lambda, \pi, \mu\right),\left(m_{0}, m\right)$ meet the conditions of Theorem 2.1. Then, if either (i) or (ii) below holds true, one has $\lambda \neq 0$ :

(i) hypothesis $(\mathbf{T Q n})_{b}$ is satisfied and the multipliers $\left(p_{0}, p, \lambda, \pi, \mu\right)$ verify the strengthened non-triviality condition (42);

(ii) hypothesis $(\mathrm{TQn})_{f}$ is satisfied and the multipliers $\left(p_{0}, p, \lambda, \pi, \mu\right)$ verify the strengthened non-triviality condition (43).

The proof of this result is postponed to the Appendix.

Remark 4.8 (Multiple state constraints) Proposition 4.3 can be easily adapted to the case with multiple state constraints $h_{i}$ for $i=1, \ldots, N$ by simply choosing $h:=h_{1} \vee \cdots \vee h_{N}$. In particular, by applying the max-rule for subdifferentials, in (TQn) $b$, (b), condition (47) can be replaced with

$$
\begin{aligned}
& \left(\sum _ { i = 1 } ^ { N } \operatorname { p r o j } _ { x } \left[0,+\infty\left[\cdot \partial^{>} h_{i}\left(\bar{y}^{0}(\bar{S}), \bar{y}(\bar{S})\right)\right)\right.\right. \\
& \cap\left(-\operatorname{proj}_{x_{2}} N_{\mathcal{T}_{0}}\left(\bar{y}^{0}(0), \bar{y}(0), \bar{y}^{0}(\bar{S}), \bar{y}(\bar{S})\right) \backslash\left\{0_{n}\right\}\right)=\emptyset,
\end{aligned}
$$

and one can require that condition (48) is satisfied for all $\left(\zeta_{t_{2}}, \zeta_{x_{2}}\right)$ in the set $\operatorname{proj}_{\left(t_{2}, x_{2}\right)} N_{\mathcal{T}_{0}}\left(\bar{y}^{0}(0), \bar{y}(0), \bar{y}^{0}(\bar{S}), \bar{y}(\bar{S})\right)+\sum_{i=1}^{N}\left[0,+\infty\left[\cdot \partial^{>} h_{i}\left(\bar{y}^{0}(\bar{S}), \bar{y}(\bar{S})\right)\right.\right.$. The other conditions can be adapted in a similar way.

From Propositions 4.2, 4.3, and Theorem 4.2 we deduce as a corollary the main result of this section.

Theorem 4.3 Assume (H0)-(H2). Consider the optimal control problem (P) and its extended sense formulation $\left(\mathrm{P}_{e}\right)$. Assume that there exists a local or global extended sense minimizer $\left(\bar{S}, \bar{\omega}^{0}, \bar{\omega}, \bar{\alpha}, \bar{y}^{0}, \bar{y}, \bar{v}\right)$ such that condition $(\mathbf{C N a})$ and either $(\mathbf{C Q n})_{b}-(\mathbf{T Q n})_{b}$ or (CQn) ${ }_{f}$-(TQn $)_{f}$ are verified. Then $\left(\bar{S}, \bar{\omega}^{0}, \bar{\omega}, \bar{\alpha}, \bar{y}^{0}, \bar{y}, \bar{v}\right)$ is a normal extremal and, in consequence of Theorem 4.1, there is no local or global infimum gap, respectively. 


\section{Some Examples}

Let us illustrate the preceding theory through some examples.

Example 5.1 Here, the absence of an infimum gap can be easily deduced from the sufficient conditions introduced in Section 4. Consider the problem

$$
\left\{\begin{array}{l}
\text { minimize } \Psi(x(1)) \\
\text { over }(x, v, u)=\left(x^{1}, x^{2}, x^{3}, v, u^{1}, u^{2}\right) \in W^{1,1}\left([0,1] ; \mathbb{R}^{3} \times \mathbb{R} \times \mathbb{R}^{2}\right) \quad \text { satisfying } \\
\frac{d x}{d t}(t)=f(x(t))+g_{1}(x(t)) \frac{d u^{1}}{d t}(t)+g_{2}(x(t)) \frac{d u^{2}}{d t}(t) \\
\frac{d v}{d t}(t)=\left|\frac{d u}{d t}(t)\right| \\
\frac{d u}{d t}(t) \in \mathcal{C}:=\mathbb{R}^{2} \quad \text { a.e. } t \in[0,1], \\
x(t) \in \Omega(t) \quad \forall t \in[0,1], \\
v(0)=0, \quad v(1) \leq 2, \quad x(0) \in \mathcal{T}_{0}^{1}, \quad x(1) \in \mathcal{T}_{0}^{2} .
\end{array}\right.
$$

in which $\Psi(x):=-x^{1}$,

$$
\begin{aligned}
& \Omega(t):=\left\{(t, x) \in \mathbb{R} \times \mathbb{R}^{3}:-1 \leq x^{1} \leq 1+t,-1 \leq x^{2} \leq 1,-1 \leq x^{3} \leq 1\right\}, \\
& \mathcal{T}_{0}^{1}:=\left\{x \in \mathbb{R}^{3}:\left(x^{1}-1\right)^{2}+\left(x^{2}\right)^{2}+\left(x^{3}\right)^{2} \leq 1 / 9, x^{1} \leq 1\right\}, \\
& \mathcal{T}_{0}^{2}:=\left\{x \in \mathbb{R}^{3}:\left(x^{1}+1\right)^{2}+\left(x^{2}\right)^{2}+\left(x^{3}\right)^{2} \leq 1, x^{1} \geq-1\right\},
\end{aligned}
$$

and

$$
g_{1}(x):=\left(\begin{array}{l}
1 \\
0 \\
0
\end{array}\right), \quad g_{2}(x):=\left(\begin{array}{c}
0 \\
-1 \\
-x^{1}
\end{array}\right), \quad f(x):=\left(\begin{array}{c}
0 \\
x^{2} x^{3} \\
0
\end{array}\right) \quad \forall x \in \mathbb{R}^{3} .
$$

The extended problem is

$$
\left\{\begin{array}{l}
\text { minimize } \Psi(y(S)) \\
\text { over } S>0,\left(y^{0}, y^{1}, y^{2}, y^{3}, v, \omega^{0}, \omega^{1}, \omega^{2}\right) \in W^{1,1}([0, S]) \quad \text { satisfying } \\
\frac{d y^{0}}{d s}(s)=\omega^{0}(s) \\
\frac{d y}{d s}(s)=f(y(s)) \omega^{0}(s)+g_{1}(y(s)) \omega^{1}(s)+g_{2}(y(s)) \omega^{2}(s) \\
\frac{d v}{d s}(s)=|\omega(s)| \\
\left(\omega^{0}, \omega\right)(s) \in \mathbf{C} \quad \text { a.e. } s \in[0, S], \\
y(s) \in \Omega\left(y^{0}(s)\right) \quad \forall s \in[0, S], \\
v(0)=0, v(S) \leq 2,\left(y^{0}(0), y(0)\right)=\{0\} \times \mathcal{T}_{0}^{1}, \quad\left(y^{0}(S), y(S)\right) \in\{1\} \times \mathcal{T}_{0}^{2} .
\end{array}\right.
$$

An extended sense minimizer is clearly given by the feasible extended sense process $\left(\bar{S}, \bar{\omega}^{0}, \bar{\omega}, \bar{y}^{0}, \bar{y}, \bar{v}\right)$, where

$$
\bar{S}=2, \quad\left(\bar{\omega}^{0}, \bar{\omega}\right)=\left(\bar{\omega}^{0}, \bar{\omega}^{1}, \bar{\omega}^{2}\right)=(1,0,0) \chi_{[0,1]}+(0,-1,0) \chi_{] 1,2]},
$$


and one considers the corresponding trajectory with initial state condition $y(0)=(1,0,0)$, namely,

$$
\left(\bar{y}^{0}, \bar{y}, \bar{v}\right)=\left(\bar{y}^{0}, \bar{y}^{1}, \bar{y}^{2}, \bar{y}^{3}, \bar{v}\right)=(s, 1,0,0,0) \chi_{[0,1]}+(1,2-s, 0,0, s-1) \chi_{[1,2]} .
$$

Indeed, for all points $\left(x^{1}, x^{2}, x^{3}\right) \in \mathcal{T}_{0}^{2}$ one has $x^{1} \in[-1,0]$, so that $\bar{y}^{1}(2)=0$ is the minimum admissible value of the cost function $\Psi(y(S))=-y^{1}(S)$. It is not difficult to check that this process verifies conditions $(\mathbf{C N a}),(\mathbf{C Q n})_{b}$, and (TQn) $)_{b}$. In consequence, the absence of a gap between the infima of problems Eqs. 53 and 54 follows directly from Theorem 4.3.

Next example shows how the criterion of normality can guarantee the absence of the infimum gap in situations where other sufficient conditions fail.

Example 5.2 Consider again the minimization problem (53) and its extended version (54), where $\mathcal{T}_{0}^{1}$ is as above, while the time-dependent state constraint $\Omega(t)$ and the final-point constraint $\mathcal{T}_{0}^{2}$ are replaced with

$$
\begin{aligned}
& \Omega:=\left\{\left(x^{1}, x^{2}, x^{3}\right):-1 \leq x^{1} \leq 1,-1 \leq x^{2} \leq 1,-1 \leq x^{3} \leq 1\right\}, \\
& \mathcal{T}_{0}^{2}:=\left\{\left(x^{1}, x^{2}, x^{3}\right):-1 \leq x^{1} \leq 0,0 \leq x^{2} \leq 1,0 \leq x^{3} \leq 1\right\},
\end{aligned}
$$

respectively. Then the extended sense process $\left(\bar{S}, \bar{\omega}^{0}, \bar{\omega}, \bar{y}^{0}, \bar{y}, \bar{v}\right)$ given by Eqs. 55 and 56 is still a (feasible) minimizer for the extended problem (54). However, as it is easy to check, now the nondegeneracy condition $(\mathbf{C N a})$ is met, but none of the conditions $(\mathbf{C Q n})_{b}$, $(\mathbf{T Q n})_{b},(\mathbf{C Q n})_{f}$, and (TQn) ${ }_{f}$ is satisfied.

From Theorem 2.1 there exist a set of multipliers $\left(p_{0}, p, \pi, \lambda, \mu\right)$ and functions $\left(m_{0}, m\right)$ with $\pi=0$, since $\nabla_{v} \Psi \equiv 0$ and $\bar{v}(2)=1<2$. Also, $m_{0} \equiv 0$ as the state constraint does not depend on time, $\mu([0,2])=\mu([0,1])$, and $m(s) \in \partial_{x}^{>} h(\bar{y}(0)) \mu$-a.e. yields $m(s)=(1,0,0) \mu$-a.e. in $[0,1]$. By the adjoint equation it follows that the path $\left(p_{0}, p\right)=$ $\left(p_{0}, p_{1}, p_{2}, p_{3}\right) \equiv\left(\bar{p}_{0}, \bar{p}_{1}, \bar{p}_{2}, \bar{p}_{3}\right)$ is constant. From the transversality conditions

$$
\begin{aligned}
& \left(p_{0}, p_{1}, p_{2}, p_{3}\right)(0) \in \mathbb{R} \times N_{\mathcal{T}_{0}^{1}}(1,0,0), \\
& -\left(q_{0}, q_{1}, q_{2}, q_{3}\right)(2) \in \lambda\{(0,-1,0,0)\}+\mathbb{R} \times N_{\mathcal{T}_{0}^{2}}(0,0,0),
\end{aligned}
$$

where $q_{0} \equiv \bar{p}_{0}$, and $q(s)=\left(\bar{p}_{1}+\mu([0,1]), \bar{p}_{2}, \bar{p}_{3}\right)$ for all $\left.\left.s \in\right] 1,2\right]$, we derive that $\bar{p}_{0} \in \mathbb{R}$, $\bar{p}_{1} \geq 0, \bar{p}_{2}=\bar{p}_{3}=0, q_{1}(2)=\bar{p}_{1}+\mu([0,1])=\lambda-\alpha_{1}$ with $\alpha_{1} \geq 0$. The maximality condition implies the relations

$$
\left.\left.\bar{p}_{0} \chi_{[0,1]}(s)=0, \quad-q_{1}(s) \chi\right] 1,2\right](s)=0,
$$

from which we deduce that $\bar{p}_{0}=0$ and $\bar{p}_{1}+\mu([0,1])=\lambda-\alpha_{1}=0$. Hence, recalling that $\bar{p}_{1} \geq 0$, we get $\bar{p}_{1}=\mu([0,1])=0, \lambda=\alpha_{1} \geq 0$. So, the strengthened nontriviality condition $\|p\|_{L^{\infty}}+\mu([0,2])+\lambda \neq 0$ implies that $\lambda \neq 0$ and this shows that $\left(\bar{S}, \bar{\omega}^{0}, \bar{\omega}, \bar{y}^{0}, \bar{y}, \bar{v}\right)$ is a normal extremal. Consequently, there is no infimum gap for the 'normality test' established in Theorem 4.1.

However, normality itself is only a sufficient condition to avoid the gap (even for systems with drift, $f$ ).

Example 5.3 Let us consider the problem in Example 5.2 where we only modify the initialpoint target $\mathcal{T}_{0}^{1}$, replacing it with $\mathcal{T}_{0}^{1}:=\{(1,0,0)\}$. Then the extended sense process 
$\left(\bar{S}, \bar{\omega}^{0}, \bar{\omega}, \bar{y}^{0}, \bar{y}, \bar{v}\right)$ introduced in Example 5.1 is obviously still admissible and minimizing, but it is easy to see that the set of degenerate multipliers $\left(p_{0}, p, \lambda, \mu\right)$ with $p_{0}=p_{2}=$ $p_{3}=0, p_{1}=-1, \mu=\delta_{\{0\}}$, and $\lambda=0$ meets all the conditions of Theorem 2.1. So, $\left(\bar{S}, \bar{\omega}^{0}, \bar{\omega}, \bar{y}^{0}, \bar{y}, \bar{v}\right)$ is an abnormal extremal. But there is no gap, because, for any $n \in \mathbb{N}$, the strict sense process $\left(\bar{S}, \omega_{n}^{0}, \omega_{n}, y_{n}^{0}, y_{n}, v_{n}\right)$, where

$$
\bar{S}=2, \quad\left(\omega_{n}^{0}, \omega_{n}\right)=\left(1-\frac{1}{n},-\frac{1}{n}, 0\right) \chi_{[0,1]}+\left(\frac{1}{n},-1+\frac{1}{n}, 0\right) \chi_{[1,2]},
$$

and $\left(y_{n}^{0}, y_{n}, v_{n}\right)$ is the corresponding trajectory of the control system in Eq. 54 with initial condition $\left(y^{0}, y, v\right)(0)=(0,1,0,0,0)$, is feasible and minimizing for the original problem, since it has cost equal to zero.

\section{Appendix}

Proof of Theorem 4.2 Thanks to Remark 4.6, when (CQn) $)_{\mathbf{b}}$ is in force the proof of Theorem 4.2 is analogous to the proof of [25, Th. 4.2], while under assumption (CQn) it requires some adaptation. For this reason, we limit ourselves to give the proof in the last case.

By standard truncation and mollification arguments, we can assume $h$ Lipschitz continuous, with Lipschitz constant $L>0$, and $f, g_{1}, \ldots, g_{m}$, and their limiting subdifferentials in $(t, x), L^{\infty}$-bounded by some constant $\tilde{M}>0$. Set $M:=(1+m) \tilde{M}$.

By assumption, the local minimizer $\left(\bar{S}, \bar{\omega}^{0}, \bar{\omega}, \bar{\alpha}, \bar{y}^{0}, \bar{y}, \bar{v}\right)$ has a set of multipliers $\left(p_{0}, p, \pi, \lambda, \mu\right)$ and some functions $\left(m_{0}, m\right)$ such that the conditions (i)-(vi) of Theorem 2.1 hold true, and verifying the strengthened non-triviality condition (34). Let us first assume that $\bar{y}^{0}(\bar{S})>\bar{y}^{0}(0)$ and suppose by contradiction that

$$
q(0)=0, \quad \lambda=0 .
$$

Set

$$
\bar{s}:=\sup \{s \in[0, \bar{S}]: \mu(] 0, s])=0\} .
$$

Observe that $\bar{s}<\bar{S}$. Indeed, if not, $\mu(] 0, \bar{S}])=0$. But in this case $q(s)=p(s)+$ $\mu(\{0\}) m(0)$, so that it is absolutely continuous and by the adjoint equation with initial condition $q(0)=0$ it follows that $q \equiv 0$. Precisely, by known properties of the convex hull of the limiting subdifferential of locally Lipschitz continuous functions (see e.g. [35, Ch. 6]), we have

$$
|q(s)| \leq \int_{0}^{s}\left|\frac{d q}{d s}(s)\right| d s \leq M \int_{0}^{s}|q(s)| d s,
$$

which implies that $q \equiv 0$ by Gronwall's Lemma. Since $\lambda=0$ by Eq. 59 , this is in contradiction with the first relation in Eq. 34. When $\bar{y}^{0}(\bar{S})=\bar{y}^{0}(0)$ and we assume by contradiction that

$$
q_{0}(0)=0, \quad q(0)=0, \quad \lambda=0,
$$

the value $\bar{s}$ defined as above is still strictly smaller than $\bar{S}$, since otherwise $\mu(] 0, \bar{S}])=0$, so that $\left(q_{0}, q\right) \equiv 0$, again by the adjoint equation. In view of Eq. 60 , this yields contradiction with the second relation in Eq. 34. Obviously, $\left(\bar{y}^{0}(\bar{s}), \bar{y}(\bar{s})\right) \in \partial \Omega$.

From now on, the proof is the same for both cases. Introduce

$$
\left(z_{0}, z\right)(s):=\left(p_{0}(s)+m_{0}(0) \mu(\{0\}), p(s)+m(0) \mu(\{0\})\right),
$$


so that, for any $s \in[0, \bar{S}[$,

$$
\begin{aligned}
\left(q_{0}(s), q(s)\right) & =\left(p_{0}(s)+\int_{[0, s[} m_{0}(\sigma) \mu(d \sigma), p(s)+\int_{[0, s[} m(\sigma) \mu(d \sigma)\right) \\
& =\left(z_{0}(s)+\int_{[0, s[} m_{0}(\sigma) \mu(d \sigma), z(s)+\int_{] 0, s[} m(\sigma) \mu(d \sigma)\right) .
\end{aligned}
$$

By the adjoint equation, $\left(z_{0}, z\right)$ verifies

$$
\left\{\begin{array}{l}
-\left(\frac{d z_{0}}{d s}, \frac{d z}{d s}\right)(s) \in \operatorname{co} \partial_{t, x}\{q(s) \cdot \bar{F}(s)\}=\operatorname{co} \partial_{t, x}\left\{z(s) \cdot \bar{F}(s)+\int_{] 0, s[} m(\sigma) \mu(d \sigma) \cdot \bar{F}(s)\right\} \\
z(0)=0, \quad\left(\text { and } z_{0}(0)=0, \text { if } \bar{y}^{0}(\bar{S})=\bar{y}^{0}(0)\right) .
\end{array}\right.
$$

Since the integral on the right hand side is identically zero in $] 0, \bar{s}[$, arguing as above we derive that $z(s)=0$ and therefore $q(s)=0$ for all $s \in[0, \bar{s}$ [, by continuity. Moreover, Gronwall's Lemma implies that $|z(s)| \leq C \mu([\bar{s}, s[)$ for all $s \in[\bar{s}, \bar{S}$ [, for some $C>0$, so that

$$
|q(s)| \leq|z(s)|+L \mu([\bar{s}, s[) \leq(C+L) \mu([\bar{s}, s[) \quad \forall s \in[\bar{s}, \bar{S}[.
$$

As a consequence of Eq. 61, for every $s \in[\bar{s}, \bar{S}]$ one gets $q(s)=z(s)+\int_{[\bar{s}, s[} m(\sigma) \mu(d \sigma)$, and Eqs. 62 and 63 imply

$$
\left|q(s)-\int_{[\bar{s}, s[} m(\sigma) \mu(d \sigma)\right| \leq M \int_{\bar{s}}^{s}|q(\sigma)| d \sigma \leq \bar{C} \mu([\bar{s}, s[)(s-\bar{s}),
$$

where $\bar{C}:=M(L+C)$. In view of (CQn) ${ }_{f}^{\prime}$ in Remark 4.4, there exist $\varepsilon, \delta>0$ and a measurable control $(\tilde{\omega}, \hat{\alpha}):[0, \bar{S}] \rightarrow(\mathcal{C} \cap \partial \mathbb{B}) \times A$, verifying for all $\left(\xi_{0}, \xi\right) \in \partial^{*} h\left(\bar{y}^{0}(s), \bar{y}(s)\right)$ with $s \in] \bar{s}, \bar{s}+\varepsilon[\cap[0, \bar{S}]$ :

$$
\xi \cdot\left(F\left(\left(\bar{y}^{0}, \bar{y}, \hat{\omega}^{0}, \hat{\omega}, \hat{\alpha}\right)\left(s^{\prime}\right)\right)-\bar{F}\left(s^{\prime}\right)\right)>\delta, \quad \text { for a.e. } s^{\prime} \in \Gamma(\bar{s}, \varepsilon),
$$

where $\left(\hat{\omega}^{0}(s), \hat{\omega}(s), \hat{\alpha}(s)\right):=\left(\bar{\omega}^{0}(s),\left(1-\bar{\omega}^{0}(s)\right) \tilde{\omega}(s), \hat{\alpha}(s)\right)$ for a.e. $s \in[0, \bar{S}]$. Observe that, being $\hat{\omega}^{0} \equiv \bar{\omega}^{0}$, one has $|\hat{\omega}|=1-\bar{\omega}^{0}=|\bar{\omega}|$ a.e. As observed in Remark 4.6, $\ell(\Gamma(\bar{s}, \varepsilon))$ is $>0$ for any $\varepsilon>0$ sufficiently small. Moreover, Eq. 65 is valid for any $\left(\xi_{0}, \xi\right) \in \partial^{C} h\left(\bar{y}^{0}(s), \bar{y}(s)\right)$, so that it is true, in particular, for $\left(\xi_{0}, \xi\right) \in \partial^{>} h\left(\bar{y}^{0}(s), \bar{y}(s)\right)$. On the other hand, by the maximization condition (11) of Theorem 2.1, it follows that, for a.e. $s \in] \bar{s}, \bar{s}+\varepsilon[\cap[0, \bar{S}]$,

$$
\begin{aligned}
& q_{0}(s)\left(\hat{\omega}^{0}(s)-\bar{\omega}^{0}(s)\right)+\pi(|\hat{\omega}(s)|-|\bar{\omega}(s)|)+q(s)\left[F\left(\left(\bar{y}^{0}, \bar{y}, \hat{\omega}^{0}, \hat{\omega}, \hat{\alpha}\right)(s)\right)-\bar{F}(s)\right] \\
& =q(s)\left[F\left(\left(\bar{y}^{0}, \bar{y}, \hat{\omega}^{0}, \hat{\omega}, \hat{\alpha}\right)(s)\right)-\bar{F}(s)\right] \leq 0 .
\end{aligned}
$$

Putting together Eqs. 64, 65, and 66 we get the desired contradiction. Indeed, for $\varepsilon>0$ small enough, for any $s^{\prime} \in \Gamma(\bar{s}, \varepsilon)$, one has

$$
\begin{aligned}
0 & \geq q\left(s^{\prime}\right)\left[F\left(\left(\bar{y}^{0}, \bar{y}, \hat{\omega}^{0}, \hat{\omega}, \hat{\alpha}\right)\left(s^{\prime}\right)\right)-\bar{F}\left(s^{\prime}\right)\right] \\
& =\left(q\left(s^{\prime}\right)-\int_{\left[\bar{s}, s^{\prime}[\right.} m(\sigma) \mu(d \sigma)+\int_{\left[\bar{s}, s^{\prime}[\right.} m(\sigma) \mu(d \sigma)\right)\left[F\left(\left(\bar{y}^{0}, \bar{y}, \hat{\omega}^{0}, \hat{\omega}, \hat{\alpha}\right)\left(s^{\prime}\right)\right)-\bar{F}\left(s^{\prime}\right)\right] \\
& \geq \int_{\left[\bar{s}, s^{\prime}[\right.} m(\sigma)\left[F\left(\left(\bar{y}^{0}, \bar{y}, \hat{\omega}^{0}, \hat{\omega}, \hat{\alpha}\right)\left(s^{\prime}\right)\right)-\bar{F}\left(s^{\prime}\right)\right] \mu(d \sigma)-2 M \bar{C} \mu\left(\left[\bar{s}, s^{\prime}[)\left(s^{\prime}-\bar{s}\right)\right.\right. \\
& \geq \mu\left(\left[\bar{s}, s^{\prime}[)\left[\delta-2 M \bar{C}\left(s^{\prime}-\bar{s}\right)\right]>0\right.\right.
\end{aligned}
$$

for $\varepsilon>0$ sufficiently small. This concludes the proof.

Proof of Proposition 4.3 The proof follows the same lines of the proof of [25, Prop. 4.1], where however only condition (TQn) $)_{\mathbf{b}}$ for an implicit state constraint $\Omega \subseteq \mathbb{R}^{n}$ is consid- 
ered. Let us prove (i). Assume by contradiction $\lambda=0$. Then the transversality condition (10) implies that

$$
\left(-q_{0}(\bar{S}),-q(\bar{S})\right)=\left(\zeta_{t_{2}}, \zeta_{x_{2}}\right) \in \operatorname{proj}_{\left(t_{2}, x_{2}\right)} N_{\mathcal{T}_{0}}\left(\bar{y}^{0}(0), \bar{y}(0), \bar{y}^{0}(\bar{S}), \bar{y}(\bar{S})\right),
$$

where $\left(\zeta_{t_{2}}, \zeta_{x_{2}}\right) \neq(0,0)$ and, in particular, $\zeta_{x_{2}} \neq 0$ if $\bar{y}^{0}(\bar{S})>\bar{y}^{0}(0)$ by Eq. 42. By hypothesis (TQn) $\mathbf{b}$, there is some $\varepsilon>0$ such that $\left(\bar{y}^{0}(s), \bar{y}(s)\right) \in \operatorname{Int}(\Omega)$ for all $s \in[\bar{S}-$ $\varepsilon, \bar{S}\left[\right.$. Hence $\mu\left([\bar{S}-\varepsilon, \bar{S}[)=0\right.$, so that, for any $s \in] \bar{S}-\varepsilon, \bar{S}\left[,\left(q_{0}, q\right)\right.$ is continuous at $s$ and

$$
\left(q_{0}(s), q(s)\right)=\left(p_{0}(s)+\int_{[0, \bar{S}-\varepsilon]} m_{0}(r) \mu(d r), p(s)+\int_{[0, \bar{S}-\varepsilon]} m(r) \mu(d r)\right) .
$$

Set $\left(q_{0}\left(\bar{S}^{-}\right), q\left(\bar{S}^{-}\right)\right):=\lim _{s \rightarrow \bar{S}^{-}}\left(q_{0}(s), q(s)\right)=\left(p_{0}, p\right)(\bar{S})+\int_{[0, \bar{S}-\varepsilon]}\left(m_{0}, m\right)(r) \mu(d r)$. We get

$$
\left(q_{0}\left(\bar{S}^{-}\right), q\left(\bar{S}^{-}\right)\right)=\left(q_{0}(\bar{S})-m_{0}(\bar{S}) \mu(\{\bar{S}\}), q(\bar{S})-m(\bar{S}) \mu(\{\bar{S}\})\right)=\left(-\tilde{\zeta}_{t_{2}},-\tilde{\zeta}_{x_{2}}\right),
$$

where $\left(\tilde{\zeta}_{t_{2}}, \tilde{\zeta}_{x_{2}}\right):=\left(\zeta_{t_{2}}+\mu(\{\bar{S}\}) m_{0}(\bar{S}), \zeta_{x_{2}}+\mu(\{\bar{S}\}) m(\bar{S})\right)$. Thus, in particular, the pair $\left(\tilde{\zeta}_{t_{2}}, \tilde{\zeta}_{x_{2}}\right)$ verifies

$$
\left(\tilde{\zeta}_{t_{2}}, \tilde{\zeta}_{x_{2}}\right) \in \operatorname{proj}_{\left(t_{2}, x_{2}\right)} N_{\mathcal{T}_{0}}\left(\bar{y}^{0}(0), \bar{y}(0), \bar{y}^{0}(\bar{S}), \bar{y}(\bar{S})\right)+\left[0,+\infty\left[\cdot \partial^{>} h\left(\bar{y}^{0}(\bar{S}), \bar{y}(\bar{S})\right) .\right.\right.
$$

The continuity of $\left(q_{0}, q\right)$ on $] \bar{S}-\varepsilon, \bar{S}$ [ also implies that the equality (11) in the Maximum Principle is verified for all $s \in] \bar{S}-\varepsilon, \bar{S}$ [. Hence, passing to the limit in it as $s$ tends to $\bar{S}^{-}$, we obtain

$$
\left(\tilde{\zeta}_{x_{2}} \cdot f\left(\bar{y}^{0}(\bar{S}), \bar{y}(\bar{S}), a\right)+\tilde{\zeta}_{t_{2}}\right) \omega^{0}+\tilde{\zeta}_{x_{2}} \cdot \sum_{j=i}^{m} g_{j}\left(\bar{y}^{0}(\bar{S}), \bar{y}(\bar{S})\right) \omega^{j}-\pi|\omega| \geq 0 .
$$

Suppose first that condition (a) in (TQn) $)_{\mathbf{b}}$ is satisfied. Then, from Eq. 45 we deduce that $\left(\tilde{\zeta}_{t_{2}}, \tilde{\zeta}_{x_{2}}\right) \neq(0,0)$ and choosing $\omega=0$ in Eq. 68 we obtain a contradiction to Eq. 46.

If instead condition (b) in (TQn) $)_{\mathbf{b}}$ is valid, $\pi=0$ and Eq. 42 implies that $\zeta_{x_{2}} \neq 0$. In view of Eq. 67 and hypothesis (47), this yields $\tilde{\zeta}_{x_{2}} \neq 0$. At this point, we get a contradiction to Eq. 48 by choosing $\omega^{0}=0$ in Eq. 68. The proof of (ii) is very similar, hence we omit it.

Funding Open access funding provided by Università degli Studi di Padova within the CRUI-CARE Agreement.

Open Access This article is licensed under a Creative Commons Attribution 4.0 International License, which permits use, sharing, adaptation, distribution and reproduction in any medium or format, as long as you give appropriate credit to the original author(s) and the source, provide a link to the Creative Commons licence, and indicate if changes were made. The images or other third party material in this article are included in the article's Creative Commons licence, unless indicated otherwise in a credit line to the material. If material is not included in the article's Creative Commons licence and your intended use is not permitted by statutory regulation or exceeds the permitted use, you will need to obtain permission directly from the copyright holder. To view a copy of this licence, visit http://creativecommonshorg/licenses/by/4.0/.

\section{References}

1. Aronna, M.S., Motta, M., Rampazzo, F.: Infimum gaps for limit solutions. Set-Valued Var. Anal. 23(1), 3-22 (2015)

2. Aronna, M.S., Rampazzo, F.: $\mathcal{L}^{1}$ limit solutions for control systems. J. Differential Equations 258, 954 979 (2015) 
3. Aronna, M.S., Motta, M., Rampazzo, F.: Necessary conditions involving Lie brackets for impulsive optimal control problems. In: Proc. of the 58th IEEE Conference on Decision and Control, CDC 2019, Nice, pp. 1474-1479 (2019)

4. Arutyunov, A.V., Aseev, S.M.: Investigation of the degeneracy phenomenon of the maximum principle for optimal control problems with state constraints. SIAM J. Control Optim. 35(3), 930-952 (1997)

5. Arutyunov, A.V.: Optimality Conditions. Abnormal and degenerate problems. Mathematics and its Applications, 526. Kluwer Academic Publishers, Dordrecht (2000)

6. Arutyunov, A.V., Karamzin, D.Y., Pereira, F.L.: A nondegenerate maximum principle for the impulse control problem with state constraints. SIAM J. Control Optim. 43(5), 1812-1843 (2005)

7. Arutyunov, A.V., Karamzin, D.Y., Pereira, F.L.: State constraints in impulsive control problems: Gamkrelidze-like conditions of optimality. J. Optim. Theory Appl. 166(2), 440-459 (2015)

8. Arutyunov, A.V., Karamzin, D.Y.: Non-degenerate necessary optimality conditions for the optimal control problem with equality-type state constraints. J. Global Optim. 64(4), 623-647 (2016)

9. Arutyunov, A.V., Karamzin, D.Y., Pereira, F.L.: Investigation of controllability and regularity conditions for state constrained problems. In: IFAC-Papersonline, Proceedings of the IFAC Congress in Toulouse, France, pp. 6295-6302 (2017)

10. Bressan, A., Rampazzo, F.: On differential systems with vector-valued impulsive controls. Boll. Un. Mat. Ital. B (7) 2(3), 641-656 (1988)

11. Ferreira, M.M.A., Vinter, R.B.: When is the maximum principle for state constrained problems nondegenerate. J. Math. Anal. Appl. 187(2), 438-467 (1994)

12. Ferreira, M.M.A., Fontes, F.A.C.C., Vinter, R.B.: Nondegenerate necessary conditions for nonconvex optimal control problems with state constraints. J. Math. Anal. Appl. 233(1), 116-129 (1999)

13. Fontes, F.A.C.C., Frankowska, H.: Normality and nondegeneracy for optimal control problems with state contraints. J. Opt. Theory. Appl. 166(1), 115-136 (2015)

14. Frankowska, H., Tonon, D.: Inward pointing trajectories, normality of the maximum principle and the non occurrence of the Lavrentieff phenomenon in optimal control under state constraints. Journal of Convex Analysis 20(4), 1147-1180 (2013)

15. Hájec, O.: Book review: differential systems involving impulses. Bull. Amer. Math. Soc. 12, 272-279 (1985)

16. Hou, S.H., Wong, K.H.: Optimal impulsive control problem with application to human immunodeficiency virus treatment. J. Optim. Theory Appl. 151, 385-401 (2011)

17. Karamazin, D.Y.: Necessary conditions for the minimum in an impulsive optimal control problem. J.Math. Sci. (N.Y.) 139(6), 7087-7150 (2006)

18. Lopes, S.O., Fontes, F.A.C.C., de Pinho, M.D.R.: On constraint qualifications for nondegenerate necessary conditions of optimality applied to optimal control problems. Discrete Contin. Dyn. Syst. 29(2), 559-575 (2011)

19. Karamzin, D.Y., de Oliveira, V.A., Pereira, F.L., Silva, G.N.: On the properness of an impulsive control extension of dynamic optimization problems. ESAIM Control Optim. Calc. Var. 21(3), 857-875 (2015)

20. Mariconda C.: Equi-Lipschitz minimizing trajectories and the Lavrentiev phenomenon for a noncoercive discontinuous non convex Bolza controlled-linear optimal control problem, to appear in Trans. Amer. Math. Soc. Accepted

21. Miller, B.M.: The method of discontinuous time substitution in problems of the optimal control of impulse and discrete-continuous systems. (Russian) Avtomat. i Telemekh. 1993, no. 12, 3-32; Translation in Automat. Remote Control 54 (1993), no. 12, part 1, pp. 1727-1750 (1994)

22. Miller, B.M., Rubinovich, E.Y.: Impulsive Control in Continuous and Discrete-Continuous Systems. Kluwer Academic/Plenum Publishers, New York (2003)

23. Motta, M., Rampazzo, F.: Space-time trajectories of non linear systems driven by ordinary and impulsive controls. Differ. Int. Eq. 8, 269-288 (1995)

24. Motta, M., Sartori, C.: On $\mathcal{L}^{1}$ limit solutions in impulsive control. Discrete Contin. Dyn. Syst. Ser. S 11, 1201-1218 (2018)

25. Motta, M., Sartori, C.: Normality and nondegeneracy of the maximum principle in optimal impulsive control under state constraints. J. Optim. Theory Appl. 185, 44-71 (2020)

26. Motta, M., Rampazzo, F., Vinter, R.B.: Normality and gap phenomena in optimal unbounded control. ESAIM Control Optim. Calc. Var 24(4), 1645-1673 (2018)

27. Palladino, M., Rampazzo, F.: A no infimum-gap criterion. In: Proceedings of the 2019 IEEE 58th Conference on Decision and Control (CDC) Palais des congrès et des Expositions Nice Acropolis Nice, France, December 11-13, pp. 535-540 (2019)

28. Palladino, M., Rampazzo, F.: A geometrically based criterion to avoid infimum-gaps in Optimal Control. J. Differential Equations 269(11), 10107-10142 (2020) 
29. Palladino, M., Vinter, R.B.: When are minimizing controls also minimizing extended controls? Discrete Continuous Dynamical System 35(9), 4573-4592 (2015)

30. Paladino, M., Vinter, R.B.: Regularity of the hamiltonian along optimal trajectories. SIAM J. Control Optim. 53(4), 1892-1919 (2015)

31. Rampazzo, F., Vinter, R.B.: A theorem on existence of neighbouring trajectories satisfying a state constraint, with applications to optimal control. IMA J. Math. Control Inform. 16(4), 335-351 (1999)

32. Rishel, R.W.: An extended Pontryagin principle for control systems whose control laws contain measures. SIAM Journal of Control 3(2), 191-205 (1965)

33. Sarychev, A.: Nonlinear systems with impulsive and generalized function controls, in: Nonlinear Synthesis, Sopron, 1989. In: Progr. Systems Control Theory, vol. 9, Birkhäuser Boston, Boston, MA, pp. 244-257 (1991)

34. Sethi, S.P., Thompson, G.L. Optimal Control Theory: Appilcations to Management Science and Economics, 2nd edn. Kluwer Academic Publishers, Boston (2000)

35. Vinter, R.B.: Optimal Control. Birkhäuser, Boston (2000)

36. Vinter, R.B.: Free end-time optimal control problems: conditions for the absence of an infimum gap. Vietnam J. Math. 47, 757-768 (2019)

37. Warga, J.: Variational problems with unbounded controls. J. Soc. Indust. Appl. Math. Ser. A Control 3, 424-438 (1965)

38. Warga, J.: Normal control problems have no minimizing strictly original solutions. Bulletin of the Amer. Math. Soc. 77(4), 625-628 (1971)

39. Warga, J.: Optimal Control of Differential and Functional Equations. Academic Press, New York (1972)

40. Warga, J.: Optimization and controllability without differentiability assumptions. SIAM J. Control Optim. 21(6), 837-855 (1983)

41. Wolenski, P., Žabić, S.: A sampling method and approximation results for impulsive systems. SIAM J. Control Optim. 46(3), 983-998 (2007)

Publisher's Note Springer Nature remains neutral with regard to jurisdictional claims in published maps and institutional affiliations. 